\title{
A finite deformation membrane based on inter-atomic potentials for the transverse mechanics of nanotubes
}

\author{
Marino Arroyo, Ted Belytschko * \\ Department of Mechanical Engineering, Northwestern University, 2145 Sheridan Road, Evanston, IL 60208, USA
}

\begin{abstract}
A finite deformation hyper elastic membrane theory based on inter atomic potentials for crystalline films composed of a single atomic layer is developed. For this purpose, an extension of the standard Born rule that exploits the dif ferential geometry concept of the exponential map is proposed to deal with the curvature of surfaces. The exponential map is approximated locally and strain measures based on the stretch and the curvature of the membrane arise. The methodology is first particularized to atomic chains in two dimensions, and then to graphene sheets. A reduced model for the transverse mechanics of carbon nanotubes is developed in detail. This model is a hyper elastic constrained membrane which fully exploits the symmetry of the transverse deformation. Additionally, a continuum version of the non bonded interactions is provided. The continuum model is discretized using finite elements and very good agreement with molecular mechanics simulations is obtained. Finally, several simulations illustrate the strong effect of the van der Waals interactions in the transverse deformation of carbon nanotubes.
\end{abstract}

Keywords: Crystal elasticity; Quasicontinuum; Born rule; Exponential map; Membrane; Carbon nanotubes

\section{Introduction}

The study of crystals by means of continuum elasticity theory is well established. It is possible to extract the elastic constants of the crystal from the force constants of the inter-atomic interactions (Martin, 1975). It is also possible extend the con-

\footnotetext{
* Corresponding author. Tel.: +1 8474914029 ; fax: +1 847 4914011.

Email addresses: m arroyo@northwestern.edu (M. Ar royo), tedbelytschko@northwestern.edu (T. Belytschko).
}

tinuum theory to the finite deformation realm. In order to formulate a hyper-elastic strain energy density from the inter-atomic potentials, a key ingredient is the Cauchy Born rule, simply called Born rule in the following (Ericksen, 1984; Zanzotto, 1996). This classical rule is a kinematic assumption that links the atomic and the continuum deformations. It states that the crystal vectors defined by two nuclei deform according to the local deformation gradient. The resulting local hyper-elastic models describe well the crystal behavior as long as the continuum deformation is nearly homogeneous in the scale of the crystal 
vectors. In addition to the extraction of elastic material tensors, these models have been used with the finite element method to solve boundary value problems, such as the nano-indentation of silicon (Tadmor et al., 1999). If the deformation is not uniform enough for the local theory to hold, mixed continuum atomistic approaches have been proposed to deal with inhomogeneities or defects (Tadmor et al., 1996; Shenoy et al., 1999).

The appeal of the approach based on the Born rule stems from the fact that it gives rise to finite deformation constitutive models based on the nano-scale physics rather than phenomenological material models. The inter-atomic potentials, based on experimental data and quantum mechanical considerations or calculations, are thus at the core of the resulting strain energy density. A local quasicontinuum has also been developed based on the tight-binding method (Tadmor et al., 1999)

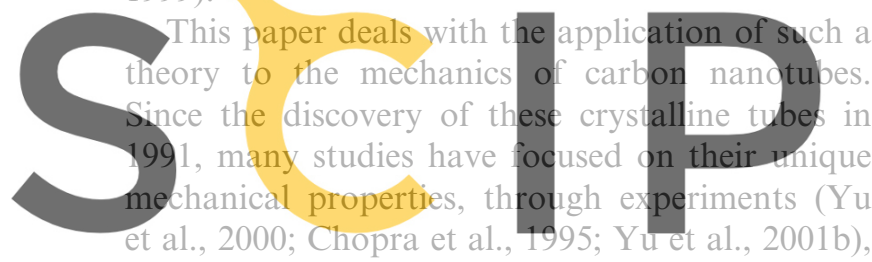

molecular dynamics (MD) and molecular me-

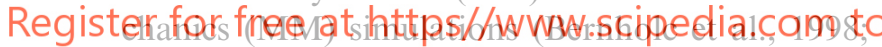

Gao et al., 1998) and first-principles calculations (Zhou et al., 2001; Maiti, 2000). Although molecular simulations seem well suited to study these systems, they are not completely satisfactory. Indeed, they are very demanding from the computational point of view. The length scales $(\AA)$ and time scales (ps) that must be resolved are often well below the scales of practical interest for a particular problem. In addition, although systems of over one million nuclei are currently being analyzed, one can always envision larger problems for which the computing capabilities do not suffice.

An approach that can alleviate some of the drawbacks of molecular simulations is the use of continuum mechanics. The ability of continuum models to describe the mechanics of nanotubes has been noted by several authors. Cross-section continuum models have been used to explain experimental observations on the transverse stability of nanotubes (Chopra et al., 1995; Yu et al., 2001a).
These extremely simplified models bring insights as well as quantitative information on the physical phenomena that govern the stability of the circular and the collapsed configurations observed in nanotubes. The vibrational properties of carbon nanotubes have been investigated through linear elasticity by Sohlberg et al. (1998). The elastic properties of carbon nanotubes as a continuum, neglecting all curvature effects, have investigated by Lu (1997). Zhong-Can et al. (1997) considered the nanotube to be an inextensible membrane, and obtained an expression of the elastic energy in terms of the curvature for a family of simple deformations. Yakobson et al. (1996) used the theory of elastic shells and linearized bifurcation analysis to study the buckling patterns of compressed carbon nanotubes observed in MD simulations. Qian et al. (2001) used a 3D continuum theory combined with a mesh-free approximation to study $\mathrm{C}_{60}$ molecules inside nanotubes.

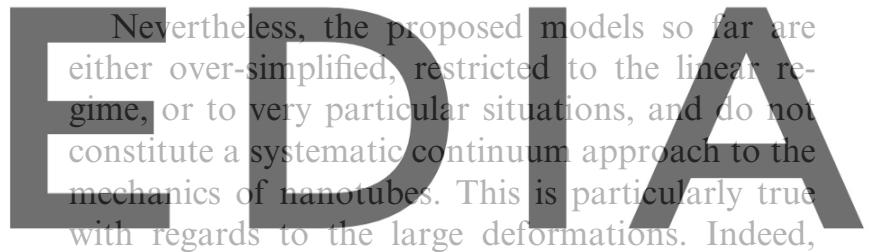

experiments (Chopra et al. 1995: Falvo et al., downgto a d therwersianiawitho utetheowatermark

1998) and first-principles calculations (Maiti, 2000) show that carbon nanotubes undergo very large deformations, with highly non-linear behavior and still remain elastic in the sense that the deformations are reversible, with stable bonds and intact bond topology.

From these considerations, developing a finite deformation model based on the Born rule and on nano-scale physics applicable to nanotubes would be of great interest and would fill in a gap in the present use of continuum models to model crystalline films one atom thick. Apart from the physical insights that a continuum model brings, such a description could be the basis for an efficient numerical simulation methodology, in contrast with the sometimes too detailed molecular simulations. Efficiency becomes an issue when nano-ropes bundles of tens to hundreds of nanotubes or multi-walled nanotubes several microns long (Ruoff et al., 1993; Yu et al., 2000) are 
to be analyzed. Furthermore, a continuum mechanics theory allows us to exploit the symmetry of certain situations explicitly, analogously to the plane strain and plane stress situations in 3D elasticity. Such reduced models for the transverse behavior of nanotubes are one of the topics presented in this paper.

Unfortunately, the traditional approach based on the Born rule works for bulk materials but fails to extend directly to the case of crystalline films and ropes one atom thick deforming in higher dimensional spaces, i.e. 3D in the case of films, and $2 \mathrm{D}$ or $3 \mathrm{D}$ in the case of ropes. The present paper describes some mechanical effects that arise from a recent extension of the Born rule to membranes (Arroyo and Belytschko, 2002). The extension is based on the differential geometry concept of the exponential map, and is here called the exponential Born rule. An alternative approach has been reported by Friesecke and James (2000).

\section{The outline of this pape}

present the Born rule for

gate its structure and expla

for films (Section 2). Then,

iminaries, Section

intro
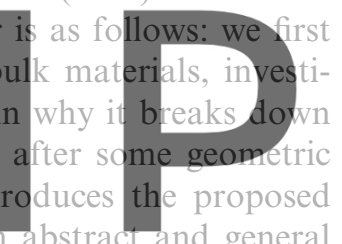

- instract and general

way. This abstract presentation of the theory is

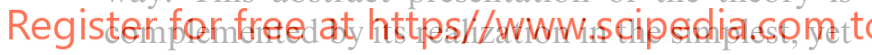

complete, situation, i.e. an atomic chain deforming in two dimensions. The formulation of the equivalent continuum rope-like object is detailed in Section 4, and a simple example illustrating the effectiveness of this model in mimicking the atomic chain is provided. Previous to the application of the theory to carbon nanotubes, their crystalline structure, as well as the instance of inter-atomic potential considered, are described in Section 5. Section 6 describes in detail the model for the transverse mechanics of carbon nanotubes. The implementation of the new theory to the arbitrary deformation of the continuum membrane in $3 \mathrm{D}$ is presented in Arroyo and Belytschko (2002). The Bravais multi-lattice nature of graphene requires the treatment of additional internal variables, the so-called inner displacements. Additionally, the van der Waals interactions are also accounted for in the continuum theory, and the continuum variational statement of the problem as well as the
Lagrangian stress measures that naturally arise are described. Finally, a validation test comparing the proposed continuum model discretized with finite elements to molecular calculations is provided in Section 7. Several simulations highlighting the relevance of the van der Waals forces in the transverse configurations of carbon nanotubes and nano-ropes are also included in this section.

\section{Breakdown of the Born rule for films}

The formulation of a finite deformation continuum model for space-filling defectless crystals based on the Born rule is relatively straightforward. The Born rule links the atomistic deformation to that of the continuum medium. Then, a representative crystallite is considered, and, for a given continuum deformation, the continuum

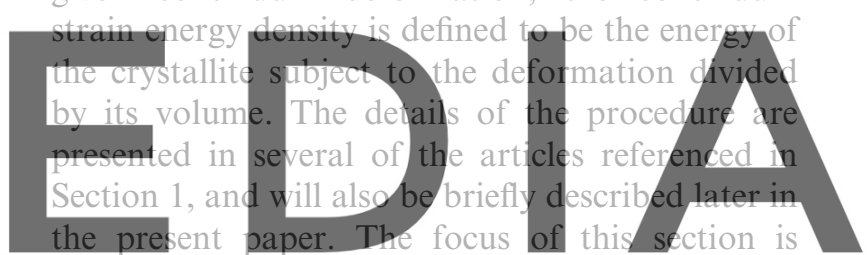

on the fundamental kinematic assumption that downsthad a the verston without the waternark 1.e. the Born rule. The details of the atomic model are deliberately omitted. Later, an instance of an atomic model is adopted.

\subsection{The standard Born rule}

Assume for the moment that we are dealing with space-filling continuum bodies, i.e. open subsets of the ambient Euclidean space. Let $\Phi$ be the deformation that maps the undeformed body $\Omega_{0} \subset \mathbb{R}^{n}$, into $\mathbb{R}^{n}, n$ being either 1,2 or 3 . If $\mathbf{X}$ denotes a point in the undeformed body, its image after deformation is $\mathbf{x}=\Phi(\mathbf{X})$. The deformed body is denoted as $\Omega=\Phi\left(\Omega_{0}\right)$ and is an open set of $\mathbb{R}^{n}$. The deformation gradient is the derivative of the vector-valued vectorial function $\Phi, \quad \mathbf{F}=$ $D \Phi=\partial \Phi / \partial \mathbf{X} \in \mathbb{R}^{n \times n}$. At each point $\mathbf{X}$, the deformation gradient is a linear transformation from $\mathbb{R}^{n}$ into $\mathbb{R}^{n}$, which maps "infinitesimal" material vectors, $\mathrm{d} \mathbf{x}=\mathbf{F} \mathrm{d} \mathbf{X}$ (see Malvern, 1969, p. 156). 
From a differential geometry point of view, the deformation gradient is called the tangent map of $\Phi$, and is denoted as $\mathbf{F}=T \Phi$. Let us call the infinitesimal neighborhoods of $\mathbf{X}$ and $\mathbf{x}$ the tangent spaces of the undeformed body and the deformed one, respectively denoted as $T_{\mathbf{X}} \Omega_{0}$ and $T_{\mathbf{x}} \Omega$ (see Fig. 1 for an illustration). Then, using this language, the deformation map $\Phi$ maps the undeformed body into the deformed one, and the tangent map $\mathbf{F}=T \Phi$ maps the tangent space of the undeformed body into the tangent space of the deformed body.

In the absence of slips, phase transitions and other special crystallographic phenomena, the Cauchy and Born hypothesis for crystals are equivalent for homogeneous deformations (Ericksen, 1984). What is referred to as the Born rule in some works is simply called the method of homogeneous deformations in others (Martin, 1975; Cousins, 1978). The Born hypothesis consists of

assuming that the lattice vectors deform as would material line elements in mation:
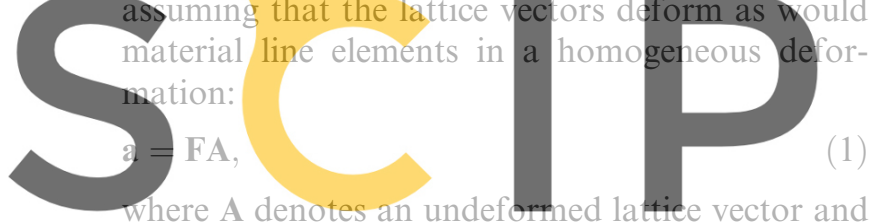

a the same vector in the deformed crystal. The

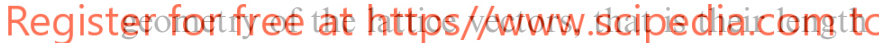
and the angles they form with other lattice vectors in the deformed crystal, can therefore be extracted from the continuum deformation through the Green deformation tensor $\mathbf{C}=\mathbf{F}^{\mathrm{T}} \mathbf{F}$ using standard continuum mechanics relations:

$\|\mathbf{a}\|=\sqrt{ } \mathbf{A} \cdot \mathbf{C A} \quad$ and $\quad \cos \theta=\frac{\mathbf{A} \cdot \mathbf{C B}}{\|\mathbf{a}\|\|\mathbf{b}\|}$,

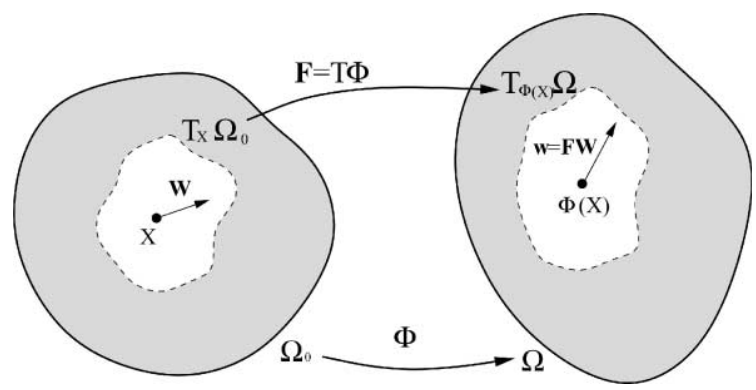

Fig. 1. Deformation map and its tangent map for space filling bodies. where $\mathbf{B}$ and $\mathbf{b}$ represent another undeformed and deformed lattice vector and $\theta$ is the angle a and $\mathbf{b}$ form in the deformed crystal. Once the geometry of the deformed lattice vectors is linked to the continuum deformation, a constitutive model based on the atomic interactions can be constructed by identifying the continuum strain energy density with the potential energy of the atomic system for a representative cell divided by its volume.

One could argue that the rule expressed by Eq. (1) is formally inconsistent, because the lattice vectors $\mathrm{A}$ and $\mathrm{a}$, each connecting two atomic positions, are physical entities that lie in the undeformed and deformed body respectively, while the tangent map $\mathbf{F}=T \Phi$ maps elements of the tangent of the undeformed body into elements of the tangent of the deformed body. This inconsistency can also be viewed from a more classic standpoint: the lattice vectors have finite length while the defor-

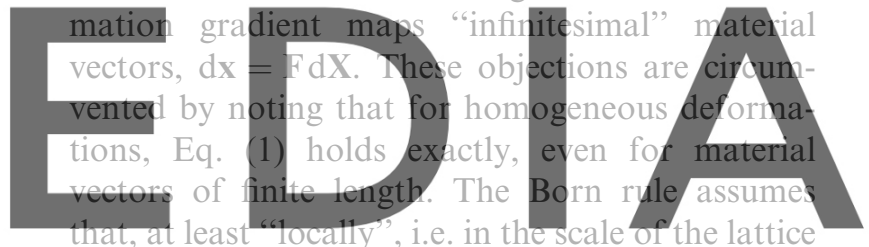

vectors, the deformation is homogeneous.

download the version without the watermark

2.2. Why the case of films is more difficult

Consider now the case in which we have a single atom thick crystalline film (such as a graphene sheet) deforming arbitrarily in 3D. It is natural in this case to treat the continuum solid as a membrane without thickness. The sheet is then a twomanifold embedded in $\mathbb{R}^{3}$ (a surface). It is assumed that the atoms lie on the surface (Cauchy's hypothesis), and therefore the lattice vectors are chords of the surface. We would like to use the Born rule in order to express the geometry of the deformed lattice vectors in terms of the some continuum variable characterizing the deformation of the surface, such as the Green deformation tensor.

Suppose that the undeformed body is planar, like a planar graphene sheet. In this case, $\Omega_{0}$ an open set in $\mathbb{R}^{2}$. The deformation map transforms this originally planar body into a curved mem- 


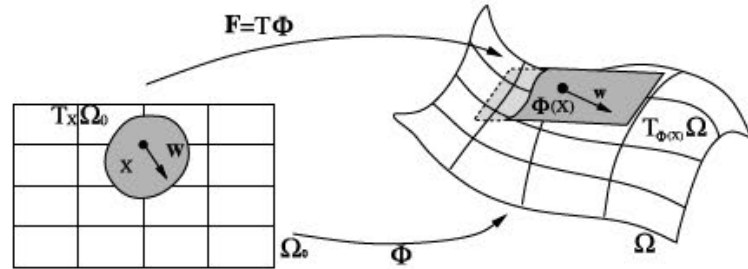

Fig. 2. Deformation map and its tangent map for surfaces in 3D.

brane, and therefore $\Omega=\Phi\left(\Omega_{0}\right)$ is now a surface in $\mathbb{R}^{3}$. The language of differential geometry introduced previously acquires a clear visual meaning in this context. Indeed, the tangent space of the deformed body at a particular point $\mathrm{x} \in \Omega, T_{\mathrm{x}} \Omega$, is the tangent plane to the surface at that point. The tangent map $\mathbf{F}=T \Phi$ maps vectors of the infinitesimal neighborhood of $\mathbf{X} \in \Omega_{0}$ into vectors tangent to the surface at $\mathrm{x}=\Phi(\mathbf{X})$, that is vectors that

belong to $T_{\mathrm{x}} \Omega$ (see Fig. 2 for an illustration). Therefore, the deformation

map cannot be used here to

$A$ in the undeformed memb

he deformed membran

Thord to the surface $\Omega$, whereas FA is tangent to

$\Omega$. The standard Born rule expressed in Eq. (1) is

Registerf for free atebttps/d wwwiscipedia.com, to

unlike the case of space-filling materials, there is

an essential distinction between the deformed

body, which is curved, and its tangent space, which is flat.

\section{An extension of the Born rule}

In this section, an extension of the standard Born rule is proposed. The formulation of the proposed kinematic assumption that links the deformation of the lattice vectors and that of the continuum body, as well as its further development in other sections, require some geometric preliminaries provided below. Then, the extension of the Born rule is described in an abstract manner, and some of its properties are sketched. We also discuss its practical application before actually implementing it for two simple instances in following sections.

\subsection{Kinematic and geometric preliminaries}

Two useful references for this section are Marsden and Hughes (1983) for a mathematical treatment of continuum mechanics, and do Carmo (1976) for the differential geometry of curves and surfaces.

For the sake of simplicity, the undeformed body is considered to be planar. This assumption simplifies the formulation, and is sufficient for the analysis of carbon nanotubes since planar graphene can be considered to be the undeformed body. For a more general presentation of the theory (see Arroyo and Belytschko, 2002). We denote the original position vector of a point and its Cartesian components by:

$$
\mathbf{X}=X^{1} \mathbf{I}_{1}+X^{2} \mathbf{I}_{2}
$$

where $\left\{\mathbf{I}_{1}, \mathbf{I}_{2}\right\}$ denotes the Cartesian basis of $\mathbb{R}^{2}$. Similarly, $\Phi$ denotes both the point mapping and

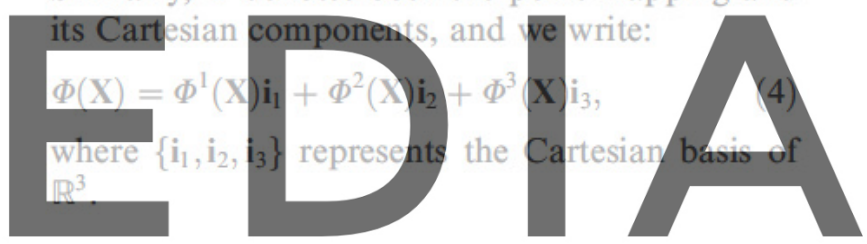

3.1.1. Metric tensor and deformation gradient download the iverision without the watermark basis vectors (tangent vectors to the surface $\Omega$ ) as:

$\mathbf{g}_{I}=\frac{\partial \tilde{\varphi}}{\partial X^{I}} \mathbf{i}_{i}$

Then, at each point $\mathbf{x},\left\{\mathbf{g}_{1}, \mathbf{g}_{2}\right\}$ is a basis of $T_{\mathbf{x}} \Omega$. We can also define the corresponding dual basis of the cotangent space of $\Omega,\left\{\mathbf{g}^{1}, \mathbf{g}^{2}\right\}$. The cotangent space $T_{\mathrm{x}}^{*} \Omega$ is the space of one-forms on $T_{\mathrm{x}} \Omega$, i.e. the linear mappings from the tangent into $\mathbb{R}$. The dual basis is defined by the relations $\mathbf{g}^{I}\left(\mathbf{g}_{J}\right)=\delta_{J}^{I}$. The metric tensor of the surface can then be defined by its covariant components (the components in the basis $\left\{\mathbf{g}^{I} \otimes \mathbf{g}^{J}\right\}$ ), which are the scalar products of the convected basis vectors:

$g_{J J}=\mathbf{g}_{I} \cdot \mathbf{g}_{J}$.

The metric tensor is actually the expression of the Euclidean scalar product in the tangent space. The tangent of the deformation map, the deformation gradient, can be expressed as: 
$\mathbf{F}=\mathbf{g}_{1} \otimes \mathbf{I}^{1}+\mathbf{g}_{2} \otimes \mathbf{I}^{2}$

where $\left\{\mathbf{I}^{1}, \mathbf{I}^{2}\right\}$ is the dual basis of $\left\{\mathbf{I}_{1}, \mathbf{I}_{2}\right\}$. Thus, a vector $\mathbf{W}=W^{I} \mathbf{I}_{I} \in T_{\mathbf{X}} \Omega_{0}$ is transformed by the deformation gradient into $\mathbf{w}=\mathbf{F W}=W^{I} \mathbf{g}_{I} \in$ $T_{\Phi(\mathbf{X})} \Omega$. Therefore, the matrix representation of $\mathbf{F}$ in the Cartesian/convected basis $\left\{\mathbf{g}_{I} \otimes \mathbf{I}^{J}\right\}$ is the $2 \times 2$ identity matrix, the information about the deformation being contained in the convected basis vectors. We can also define the Green deformation tensor $\mathbf{C}=\mathbf{F}^{\mathrm{T}} \mathbf{F}$. Its components in the Cartesian basis of $\Omega_{0}$ coincide with these of the metric tensor in the convected basis presented in Eq. (6).

The tensor $\mathbf{C}$ can be used to measure length, angle and area changes due to the deformation in terms of undeformed body quantities, i.e. C operates in $T \Omega_{0}$. In particular the element of area of $\Omega$ can be written in terms of the element of area of $\Omega_{0}$ as $\mathrm{d} \Omega=J \mathrm{~d} \Omega_{0}$ where the Jacobian is $J=$

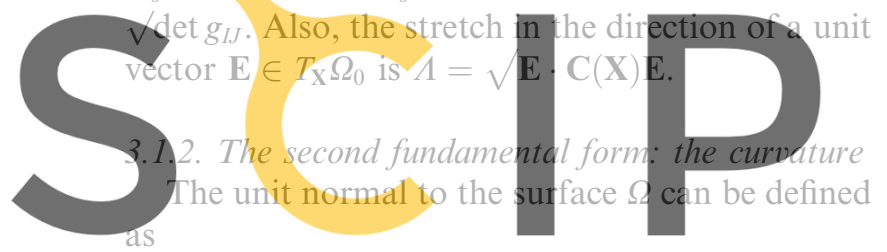

Register $\bar{f} \frac{g_{1} \times g_{1}}{f \times g_{2}}$ at at https//www.scipedia.com ${ }^{8}$ to

where $\|\cdot\|$ denotes the Euclidean norm. The second fundamental form of the deformed body $\mathbf{k}$ can be expressed in the basis $\left\{\mathbf{g}^{I} \otimes \mathbf{g}^{J}\right\}$ in terms of its components:

$k_{I J}=\mathbf{n} \cdot \mathbf{g}_{I J J}$,

where $\mathbf{g}_{I J J}$ denotes the derivative of $\mathbf{g}_{I}$ with respect to $X^{J}$. The normal curvature $k_{n}$ at a point $\mathbf{x}$ of the surface $\Omega$ and in a given direction defined by the unit vector $\mathbf{v}=v^{I} \mathbf{g}_{I} \in T_{\mathbf{x}} \Omega$, is the minimum of the curvatures of all the curves of $\Omega$ passing through $\mathbf{x}$ tangent to $\mathbf{v}$. It can be obtained as:

$k_{n}(\mathbf{x})=k_{I J} v^{I} v^{J}$.

Suppose the normal curvature of the deformed body is to be computed at a point $\mathbf{x}=\Phi(\mathbf{X}) \in \Omega$ in a given direction $\mathbf{V}=V^{I} \mathbf{I}_{I} \in T_{\mathbf{X}} \Omega_{0}$ of the undeformed body. This direction corresponds in the deformed body to $\mathbf{v}=\mathbf{F V}=V^{I} \mathbf{g}_{I}$, where Eq. (7) has been used. Therefore, after normalizing $\mathbf{v}$, the resulting expression for the normal curvature is:

$k_{n}(\mathbf{x})=\frac{k_{I J} V^{I} V^{J}}{\sqrt{ } g_{M N} V^{M} V^{N}}$,

where the denominator corresponds to the $\mathrm{Eu}-$ clidean norm of $\mathbf{v}$.

\subsubsection{The exponential map}

A simple definition of the exponential map is given in Morgan (1993) for a manifold $M$ :

\section{The exponential map exp at a point $p$ in $M$ maps the tangent space $T_{p} M$ into $M$ by send- ing a vector $\mathbf{v}$ in $T_{p} M$ to the point in $M$ a dis- tance $|\mathbf{v}|$ along the geodesic from $p$ in the direction $\mathbf{v}$.}

The exponential map is invertible and differentiable in a neighborhood of each regular point $p$ of

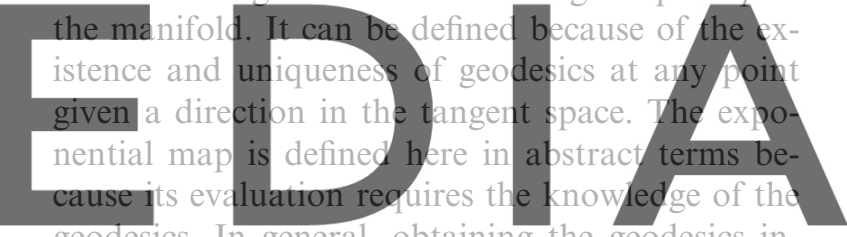

geodesics. In general, obtaining the geodesics involves solving the geodesic differential equations. downlead thenkersign wythodt the imeatermark

nary differential equations whose unknowns are the parametric coordinates of the geodesic, and whose coefficients are the Christoffel symbols of the surface. Finding the geodesics, and thus the exponential map, is much simpler in some particular cases, as will be shown for the cylinder. More details about the exponential map for surfaces can be found in do Carmo (1976). Fig. 3 provides an illustration of how the exponential map brings a tangent vector to the surface.

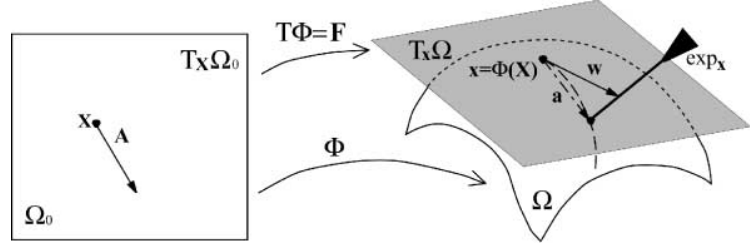

Fig. 3. Illustration of the exponential Born rule; the geodesic at $\mathbf{x}$ in the direction of $\mathbf{w}$ is represented by a dashed line. 


\subsection{Exponential Born rule}

In the present theory, the continuum solid equivalent to the original single layer crystalline film is a membrane without thickness. The nuclei of the atomistic system lie on this surface and consequently the lattice vectors are chords of the surface. Let A denote an undeformed lattice vector. In the present setting, since the undeformed body is planar, $\Omega_{0}$ and $T \Omega_{0}$ can be identified, and consequently A can be transformed through the deformation gradient. The result of the transformation $\mathbf{w}=\mathbf{F A}$ is simply the deformed lattice vector we would obtain through the standard Born rule. However the vector $\mathbf{w}$ is tangent to the deformed surface $\Omega$, not a chord. Consider the following generalized kinematic rule, called the exponential Born rule in the following:

$\mathrm{a}:=\exp _{\Phi(\mathbf{X})} \circ \mathbf{F A}$.

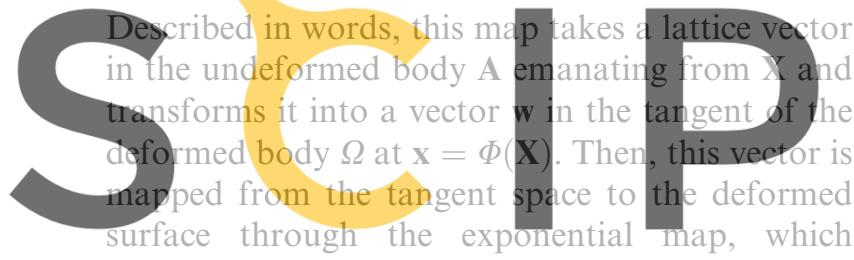

"brings" the result of the standard Born rule back

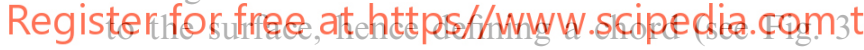

for an illustration of this procedure). Thus, the exponential Born rule links the deformation of the lattice vectors to the deformation of the continuum object, since both the deformation gradient and the exponential map are defined in terms of the deformation map $\Phi$.

This extended kinematic rule provides a theoretical framework for the application of crystal elasticity to curved crystals, by rectifying the shortcomings of the standard Born rule. Note however that its practical implementation is not straightforward, since the evaluation of the exponential map requires the determination of the geodesics, which in general entails the integration of a system of two non-linear differential equations. This results in a computationally very complex method that is necessarily non-local. Here we present approximations to the exponential map that render the model local and computationally feasible.

\section{Atomic chain in $2 \mathrm{D}$}

In this section, we illustrate the exponential Born rule for the simplest case, an atomic chain deforming in 2D. The resulting continuum model is a hyper-elastic rope whose strain energy density depends on the stretch and the curvature of the continuum object. This constitutive model is based exclusively on the atomistic description of the chain. In this case the exponential map is approximated at each point by the exponential map of the circle, for which a closed-form expression is straightforward.

\subsection{Atomic model}

The strain energy of the atomic system is described by means of bond stretch $\mathscr{V}_{s}$ and bond angle $\mathscr{V}_{\theta}$ potentials. The strain potential energy of the atomic chain can be written as a function of

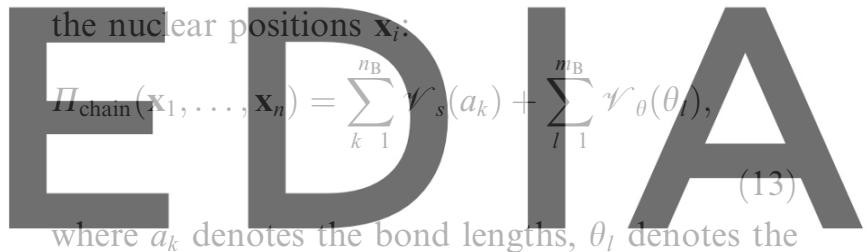

angle that adjacent bonds form, and $n_{\mathrm{B}}$ and $m_{\mathrm{B}}$ are

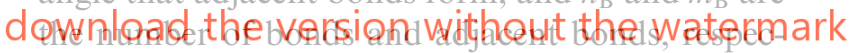

tively. This particular atomistic model is chosen for simplicity, but the approach is not restricted to this structure of the inter-atomic potential by any means. The exponential Born rule provides a link between the atomistic and the continuum deformations, and can be combined with any atomistic model of choice, not restricted to closest-neighbor models.

\subsection{Continuum model}

As illustrated in Fig. 4, the undeformed body is considered to be a 1D line segment that is allowed to deform in 2D. Therefore, the deformation map can be described as $\mathbf{x}=\Phi(X)=\Phi^{1}(X) \mathbf{i}_{1}+\Phi^{2}(X) \mathbf{i}_{2}$ with $X \in \Omega_{0} \subset \mathbb{R}$ and $\left\{\mathbf{i}_{1}, \mathbf{i}_{2}\right\}$ the basis of $\mathbb{R}^{2}$. In this case, the components of the deformation gradient are $[\mathbf{F}]=\left[\Phi_{X}^{1}, \Phi_{X}^{2}\right]^{\mathrm{T}}$, and the Green deformation tensor $C$ is a scalar, whose square root is the stretch $\Lambda$ of the deformed rope: 


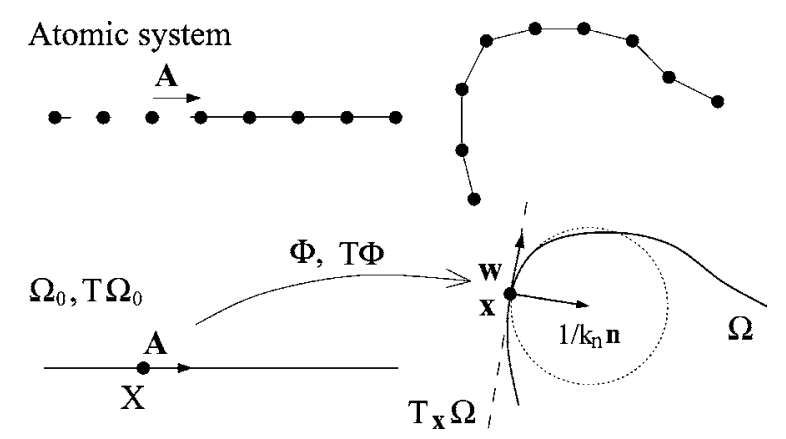

Fig. 4. Illustration of the continuum rope like model for an atomic chain deforming in $2 \mathrm{D}$.

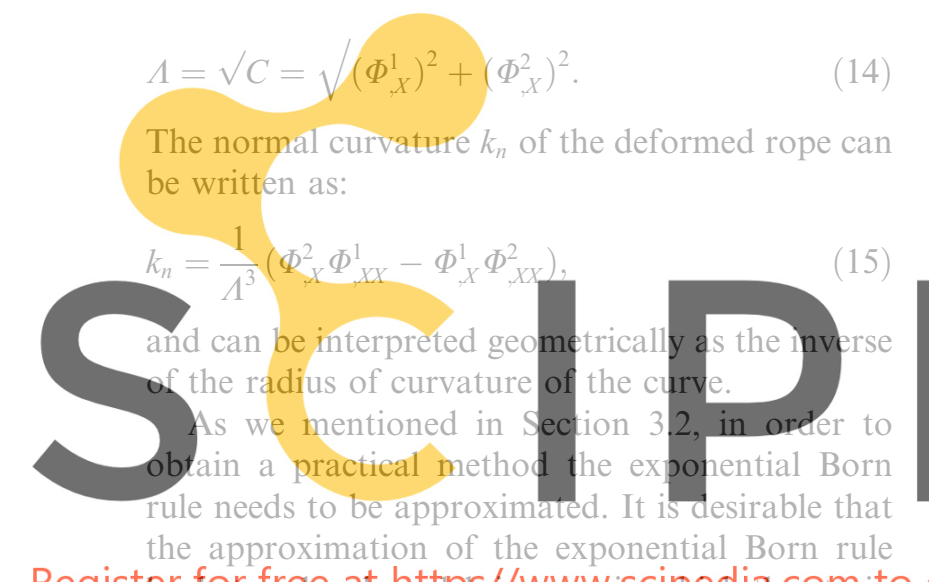

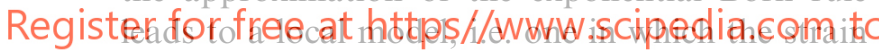

energy depends on the local deformation of the rope. The strategy followed to obtain such an approximation is to perform the exponential map at each point, not of the original curve, but of a circle of radius $r=1 / k_{n}$ with the same normal as the original curve (see Fig. 4). Thus, locally, this circle replaces the original curve. The exponential map of the circle is readily available in closed form.

The first part of the exponential Born rule maps the lattice vector A of length $A$ into a vector tangent to the curve whose components are $[\mathbf{w}]=A[\mathbf{F}]$. Therefore, its length is

$w=\sqrt{ } C A$.

The exponential map of the circle is illustrated in Fig. 5. The length of the tangent vector $\mathbf{w}$ is "walked" on the geodesic to obtain $\exp _{\Phi(\mathbf{X})} \mathbf{w}$, and therefore the chord a. Since the geodesic of the circle is trivially the circle itself, the length of the arc defined by the ends of $\mathbf{a}$ is $w$.

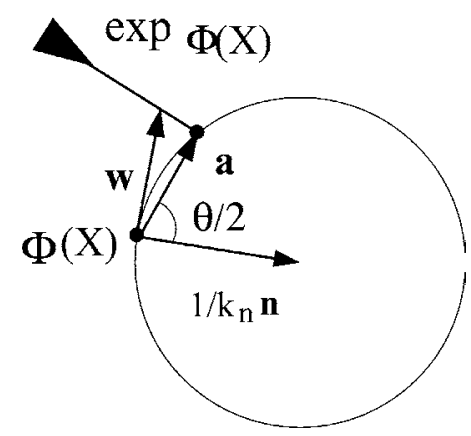

Fig. 5. The exponential map of the circle defined at each point of the curve by the unit normal and the normal curvature.

Let $\theta$ denote the angle formed by two adjacent deformed lattice vectors. Consider the triangle formed by the ends of a and the center of the circle. This triangle is isosceles, and its equal angles are $\theta / 2$. Therefore its third angle, the angle subtended by the arc of length $w$, is $\gamma=\pi-\theta$. Con-

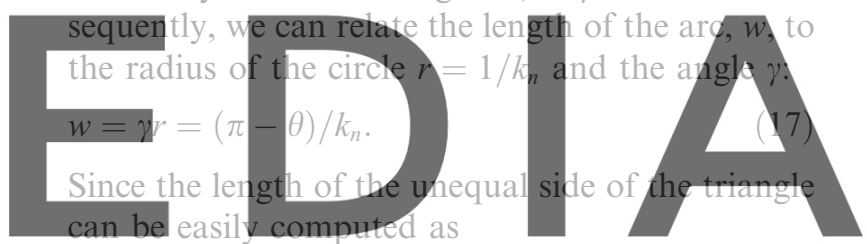

download $\overline{\bar{C}}$ the $r \sin \frac{\gamma}{\operatorname{er}}$ rsion without the watermark

it follows that

$a=\frac{2}{k_{n}} \sin \frac{k_{n} w}{2} \quad$ and $\quad \theta=\pi-k_{n} w$.

Note from Eqs. (16) and (19) that the quantities $a$ and $\theta$, which are the arguments of the atomistic energy (see Eq. (13)), are expressed in terms of the continuum deformation.

The next step is to consider a representative crystallite of the atomistic system, which in this case is a cell of length $A$ including a single nucleus in the undeformed crystal. In a homogenization process, the energy of this deformed cell containing one bond and one angle between adjacent bonds is identified to the strain energy density of the continuum multiplied by the undeformed volume of the cell: $A \cdot W(\Phi)=\mathscr{V}_{s}(a)+\mathscr{V}_{\theta}(\theta)$. Since our aim is to formulate a hyper-elastic continuum model, the elastic potential $W(\Phi)$ is a strain energy per undeformed volume, in this case undeformed 
length. The continuum strain energy density depends on the deformation map $\Phi$ through the local strain measures $C$ and $k_{n}$. Therefore, the hyperelastic potential of the continuum rope can be written as:

$$
\begin{aligned}
W\left(C, k_{n}\right)=\frac{1}{A} & \left\{\mathscr{V}_{s}\left[2 / k_{n} \sin \left(k_{n} \sqrt{ } C A / 2\right)\right]\right. \\
& \left.+\mathscr{V}_{\theta}\left[\pi-k_{n} \sqrt{ } C A\right]\right\} .
\end{aligned}
$$

The total strain energy of the continuum system approximating the atomistic energy of Eq. (13) can then be written as:

$$
\Pi_{\text {rope }}(\Phi)=\int_{\Omega_{0}} W\left(C, k_{n}\right) \mathrm{d} \Omega_{0} .
$$

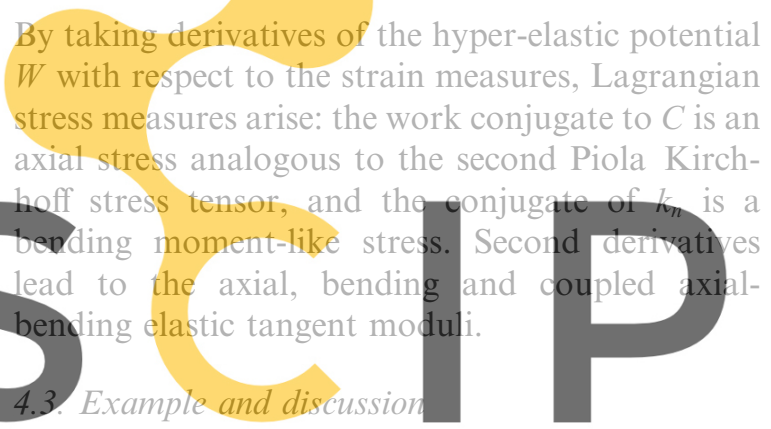

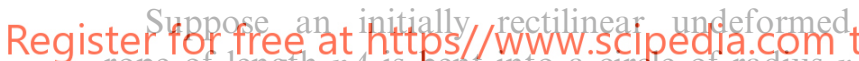
rope of length $n A$ is bent into a circle of radius $r$ with uniform stretch. The continuum stretch is $\Lambda=2 \pi r /(n A)$, and the curvature is $k_{n}=1 / r$. Since the atoms are postulated to lie on the continuum surface, the corresponding equispaced atomic chain containing $n$ bonds is deformed into a regular polygon of $n$ sides whose circumcircle has a radius $r$. The bond length and angle predicted by the continuum model (see Eqs. (16) and (19)) are:

$a=2 r \sin \frac{\pi}{n}$ and $\theta=\pi-2 \pi / n$.

It is easy to see that these predictions coincide exactly with the actual bond lengths and angles of the atomic chain deformed into a regular polygon. Therefore, the predicted energetics for this finite deformation are also exact. Of course, for a general deformation with non-constant stretch and curvature, the local approximation of the exponential Born rule will lead to approximate ener- getics. The examples presented later demonstrate, however, that this approximation is very accurate.

The inadequacy of the standard Born rule can be illustrated easily in the present example. The standard Born rule corresponds to taking $\mathbf{a}=$ $\mathbf{w}=$ FA. Suppose that our rectilinear 1D undeformed body is deformed into a circle without stretch, i.e. $\Lambda=C=1$. The application of the standard Born rule leads to deformed lattice vectors that are tangent to the rope. Consequently, two lattice vectors emanating from the same nucleus remain collinear after deformation, so the angle they form is unchanged irrespective of the bending of the rope. Furthermore, since the rope is bent without stretch, the length of the deformed lattice vectors also remains unchanged (see Eq. (16)). Therefore, the energy of such a model will remain unchanged, and the resulting rope has zero bending stiffness.

However, the real lattice vectors do not remain

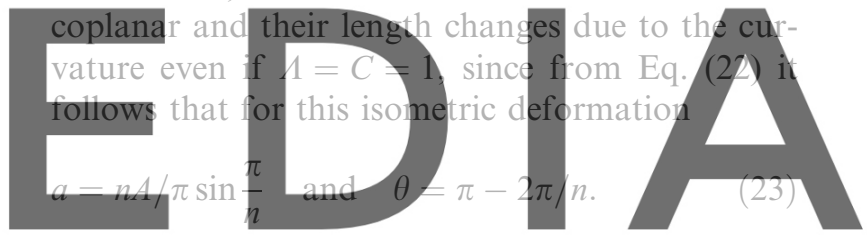

Therefore, the energy of the atomic system will down geach theldersionitwithoutsthe. Watermark continuum model based on the standard Born rule is blind to the fact that the rope is being bent, and assigns zero energy change to the deformation, in sharp contrast with the exponential Born rule, which predicts the correct energetics.

Although an intuitive approach would associate the continuum stretch to the stretch of the bonds, and the continuum curvature to changes in bond angles, the proposed model couples these deformation modes. Indeed, the continuum bond length $a$, which is the argument for the inter-atomic stretch potential, depends both on $C$ and $k_{n}$ in a non-linear fashion. The same applies to the continuum bond angle $\theta$. This feature is essential and makes the continuum model exact for deformations that map an initially straight chain into a circular arc with constant stretch. Thus, as in the case of the standard Born rule for bulk crystalline materials, the resulting continuum model for the rope is exact for homogeneous deformations. 


\section{Atomistic description of carbon nanotubes}

\subsection{Crystal structure}

The undeformed crystal is considered to be the planar graphene sheet, which upon appropriate rolling becomes a carbon nanotube (Saito et al., 1992). This choice is very natural because the interatomic potentials usually used for nanotubes have their minimum in the planar configuration; furthermore, the planar configuration facilitates the description of the crystalline structure.

The honeycomb lattice of graphene is a Bravais multi-lattice, also called a non-primitive or composite lattice. A Bravais multi-lattice can be seen as a collection of inter-penetrating simple Bravais lattices, in the case of graphene, of two simple lattices. The atomic sites can be obtained from

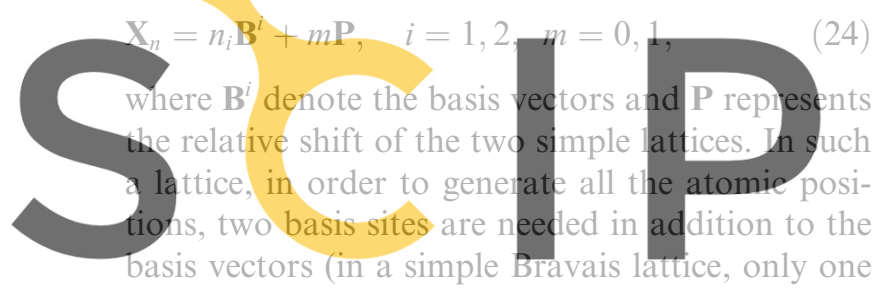
site is needed). Fig. 6 illustrates the multi-lattice

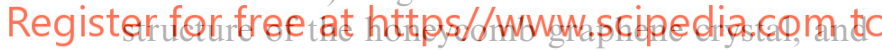
provides the expressions for the bonds of the lattice in terms of the basis vectors and the shift vector. Note that one atomic site, say a black one, and the basis vectors $\mathbf{B}^{1}$ and $\mathbf{B}^{2}$ are not enough to construct the whole lattice, in particular the white sites. In addition to that, either a white site or the shift vector $\mathbf{P}$ is needed.

Suppose the shift vector is perturbed by an inner displacement $\boldsymbol{\eta}$. Then, as can be seen from Fig. 6, the bonds of the crystal are also perturbed by the same amount, and the energy of the perturbed crystal is also changed. Note that although the crystal is changed, neither of the simple lattices deforms due to $\boldsymbol{\eta}$. It is clear that such an internal arrangement of the crystal cannot be captured by a uniform deformation such as that introduced by the Born rule. This fact has long been recognized (Cousins, 1978; Weiner, 1983; Zanzotto, 1996), and in classical crystal elasticity theory, the Born rule is postulated to affect the basis vectors, while

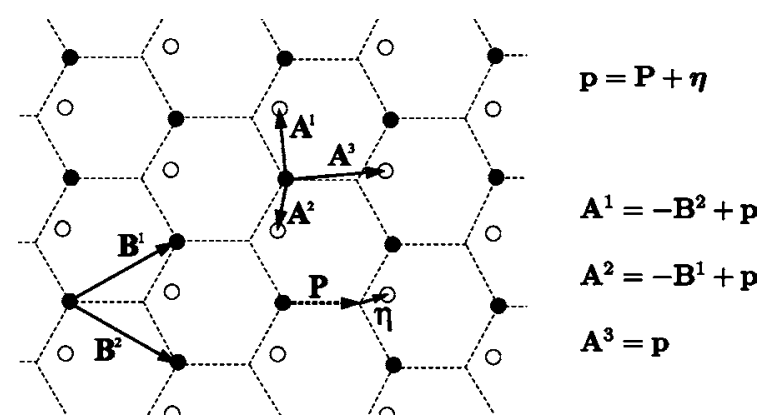

Fig. 6. Honeycomb Bravais multi lattice: basis vectors $\mathbf{B}^{i}$, shift vector $\mathbf{P}$, inner displacements $\boldsymbol{\eta}$ and bond vectors $\mathbf{A}^{k}$.

the evolution of the shift vectors is viewed as an internal rearrangement. This microstructure is obtained by minimizing the energy with respect to the shift vectors, for each given macroscopic continuum deformation. Following Tadmor et al.

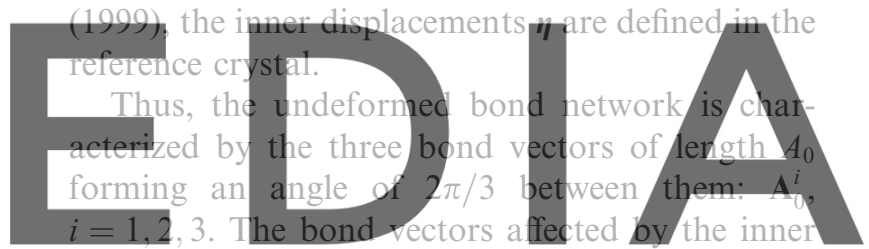

displacements are (see Fig. 6)

download the version without the watermark

Let also $A^{i}=\left\|\mathbb{A}^{i}\right\|$ and consider the three unit vectors

$\mathbf{E}^{i}=\frac{1}{A^{i}} \mathbf{A}^{i}, \quad i=1,2,3$.

The relative orientation of the original bond vectors $\mathbf{A}_{0}^{i}$ with respect to the rolling direction determines the chirality of the nanotube. For instance, one of these vectors being parallel to the nanotube axis gives rise to a zigzag nanotube. On the other hand, when one is perpendicular to the axis we have an arm-chair nanotube.

\subsection{Atomistic model}

In the following, a particular instance of atomic model is considered. Our interest here is to model covalently bonded systems, and it is assumed that 
the topology of the bond network does not change, i.e. there is no bond breaking or formation. For such a system, the energy can be accurately expressed in terms of the covalent bonds geometry, i.e. their length and the angles concurrent bonds form:

$E=E\left(r_{i}, \theta_{j}\right)$,

where $r_{i}$ denotes the lengths of the covalent bonds and $\theta_{j}$ the angles of concurrent bonds. Such a model considers only two-body/three-body interactions. However, the present theory is applicable to any atomistic model consistent with the Born Oppenheimer approximation, i.e. where the energy can be obtained in terms the nuclear positions exclusively.

Consider for example a two-body/three-body expansion of the potential energy:

$E=\sum_{i} \mathscr{V}_{s}\left(r_{i}\right)+\sum_{k} \mathscr{V}_{\theta}\left(\theta_{k}, r_{k}^{1}, r_{k}^{2}\right)$,

where the first sum runs over the covalent bonds, the second over the angles formed by covalent bonds. Here, $r_{k}^{1}$ and $r_{k}^{2}$ denote the lengths of the bonds that form the angle $\theta_{k}$. The MM models (Nevins et al., 1996) fall within this formalism. Another instance of empirical potentials dependent on bond lengths and angles are bond order potentials, such as the potentials for hydrocarbons proposed by Brenner (1990):

$E=\sum_{i}\left[\mathscr{V}_{R}\left(r_{i}\right)-b_{i}\left(r_{i}^{j}, \theta_{i}^{j}\right) \mathscr{V}_{A}\left(r_{i}\right)\right]$,

where the sum is over the bonds, and the bond order function $b_{i}$ depends on the lengths of all the bonds connected to the $i$ th bond, $r_{i}^{j}$, and on the angles they form with the $i$ th bond, $\theta_{i}^{j}$. For simplicity, in the following a two-body/three-body potential is adopted.

For these atomic potentials in which the potential energy at a nuclei or bond depends on the lengths and angles directly adjacent to it, closed form expressions for the continuum strain energy density can be obtained. The predominant short-range interactions of graphene also support the use of a local continuum model. This contrasts with the situation in other works (Tadmor et al.,
1996) where materials with longer range interactions are considered.

\section{Membrane theory for the transverse mechanics of carbon nanotubes}

In this section, a reduced model for the transverse deformation of carbon nanotubes is developed. This continuum formulation models 3D nanotubes under uniform deformation in the axial direction, i.e. allowing only transversal deformation and uniform axial stretch. In this case, the exponential map is approximated by the exponential map of the cylinder. Additionally, a continuum version of van der Waals interactions, also based on the atomistic description, is formulated.

Carbon nanotubes have very different longitudinal and transverse behavior. In the transverse direction, they are very flexible, and the effect of van der Waals interactions becomes crucial. There are many situations in which the transverse behavior is of interest, as for example in the study of the transverse stability of single-walled or multiwalled nanotubes, their interaction with substrates, or the structure of nanoropes. A reduced model for nanotubes may also be useful for studying the properties of the recently synthesized crystals of nanotubes ordered arrays of nanotubes with identical diameter and chirality on the micron scale (Schlittler et al., 2001). Thus, apart from being a simple realization of the proposed extension of classical crystal elasticity to membranes, the reduced model presented here is of interest in its own right.

\subsection{Kinematics}

We consider deformations that are uniform along the axis of the tube. These deformations can be written as:

$$
\begin{aligned}
\Phi(\mathbf{X}) & =\Phi\left(X^{1}, X^{2}\right) \\
& =\left[\Lambda_{1} X^{1}, \Phi^{2}\left(X^{2}\right), \Phi^{3}\left(X^{2}\right)\right]^{\mathrm{T}},
\end{aligned}
$$

where the $x_{1}$ axis is parallel to the tube axis (see Fig. 7). Along the $X_{1}$ axis, $\Lambda_{1}$ denotes the uniform stretch. The Cartesian bases $\mathscr{B}_{2}=\left\{\mathbf{I}_{1}, \mathbf{I}_{2}\right\}$ and $\mathscr{B}_{3}=\left\{\mathbf{i}_{1}, \mathbf{i}_{2}, \mathbf{i}_{3}\right\}$ of $\mathbb{R}^{2}$ and $\mathbb{R}^{3}$ are used. 


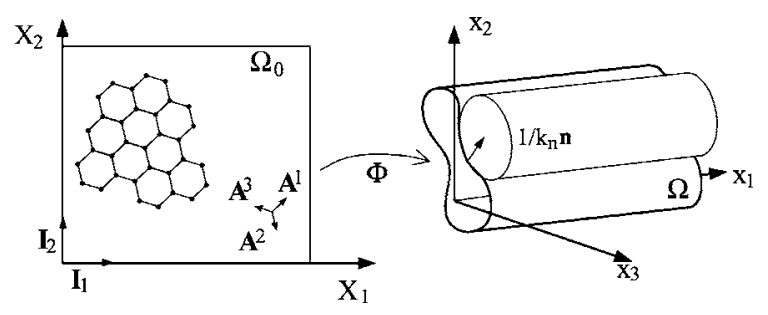

Fig. 7. Illustration of the kinematics of the nanotube with uniform deformation in the axial direction, and the cylinder used to approximate the exponential map.

\subsubsection{First fundamental form}

The convected basis vectors are:

$$
\begin{array}{ll}
\mathbf{g}_{1}=\frac{\partial \Phi}{\partial X^{1}} ; & {\left[\mathbf{g}_{1}\right]_{\mathscr{B}_{3}}=\left[\Lambda_{1}, 0,0\right]^{\mathrm{T}},} \\
\mathbf{g}_{2}=\frac{\partial \Phi}{\partial X^{2}} ; & {\left[\mathbf{g}_{2}\right]_{\mathscr{B}_{3}}=\left[0,\left(\Phi^{2}\right)^{\prime},\left(\Phi^{3}\right)^{\prime}\right]^{\mathrm{T}} .}
\end{array}
$$

The covariant components of the first fundamental form are therefore:

$$
\begin{aligned}
& g_{11}=\left(\Lambda_{1}\right)^{2} ; \quad g_{12}=g_{21}=0 ; \\
& g_{22}=\left[\left(\Phi^{2}\right)^{\prime}\right]^{2}+\left[\left(\Phi^{3}\right)^{\prime}\right]^{2} .
\end{aligned}
$$

For convenience, we will use the notation $g_{22}=$ $\left(\Lambda_{2}\right)^{2}$, where $\Lambda_{2}$ can be viewed as a circumferential stretch. Therefore, the components of the Green deformation tensor are:

$$
[\mathbf{C}]_{\mathscr{B}_{2}}=\left[\begin{array}{cc}
\left(\Lambda_{1}\right)^{2} & 0 \\
0 & \left(\Lambda_{2}\right)^{2}
\end{array}\right] \text {. }
$$

The deformation gradient can be written as:

$\mathbf{F}=\mathbf{g}_{1} \otimes \mathbf{I}^{1}+\mathbf{g}_{2} \otimes \mathbf{I}^{2}$.

Therefore, a deformed tangent vector (before the exponential map is applied) can be written as:

$\mathbf{w}=\mathbf{F A}=A \mathbf{F E}=A\left(E^{1} \mathbf{g}_{1}+E^{2} \mathbf{g}_{2}\right)$,

where $E^{1}$ and $E^{2}$ are the components in the basis $\left\{\mathbf{I}_{1}, \mathbf{I}_{2}\right\}$ of the unit vector $\mathbf{E}$ in the direction of $\mathbf{A}$, see Eq. (26). The stretch in the direction of $\mathbf{E}$ can be written as:

$\Lambda=\sqrt{ }\left(\Lambda_{1}\right)^{2}\left(E^{1}\right)^{2}+\left(\Lambda_{2}\right)^{2}\left(E^{2}\right)^{2}$.

\subsubsection{Second fundamental form}

It can be easily seen that the only non-zero covariant component of the second fundamental form is $k_{22}$ :

$$
\begin{aligned}
k_{22}= & \frac{1}{\sqrt{ }\left[\left(\Phi^{2}\right)^{\prime}\right]^{2}+\left[\left(\Phi^{3}\right)^{\prime}\right]^{2}} \\
& \times\left[-\left(\Phi^{3}\right)^{\prime}\left(\Phi^{2}\right)^{\prime \prime}+\left(\Phi^{2}\right)^{\prime}\left(\Phi^{3}\right)^{\prime \prime}\right] .
\end{aligned}
$$

The normal curvature in the circumferential direction (normal to the $X_{1}$ axis) is:

$$
\begin{aligned}
k_{n}= & \frac{1}{\left\{\left[\left(\Phi^{2}\right)^{\prime}\right]^{2}+\left[\left(\Phi^{3}\right)^{\prime}\right]^{2}\right\}^{3 / 2}} \\
& \times\left[-\left(\Phi^{3}\right)^{\prime}\left(\Phi^{2}\right)^{\prime \prime}+\left(\Phi^{2}\right)^{\prime}\left(\Phi^{3}\right)^{\prime \prime}\right] .
\end{aligned}
$$

Note that $1 / k_{n}$ is the local radius of curvature of the cylindrical surface and that the principal curvatures of the surface are $k_{n}$ and 0 .

\subsubsection{Exponential map}

In this case, the exponential map in the deformed body is approximated by the exponential map in the cylinder defined by the unit normal, the normal curvature $k_{n}$, and whose axis is parallel to the $x_{1}$-axis (see Fig. 7).

Let us now obtain an expression for the image through the exponential map of a tangent vector $\mathbf{w}=\mathbf{F A}$, and also an expression for the final deformed bond a. Consider the coordinate system $(u, v)$ for the tangent space at each point $\Phi(\mathbf{X})$ (see Fig. 8). This system is such that the $u$-axis is parallel to the $x_{1}$-axis. The length of the tangent vector $\mathbf{w}$ is $A \Lambda$ and the angle $\varphi$ it forms with the $u$-axis follows from standard continuum mechanics relations:

$$
\begin{aligned}
& \cos \varphi=\frac{1}{\Lambda \Lambda_{1}} \mathbf{E} \cdot \mathbf{C I}_{1}=\frac{\Lambda_{1}}{\Lambda} E^{1}, \\
& \sin \varphi=\frac{1}{\Lambda \Lambda_{2}} \mathbf{E} \cdot \mathbf{C} \mathbf{I}_{2}=\frac{\Lambda_{2}}{\Lambda} E^{2} .
\end{aligned}
$$

In order to obtain a closed-form expression for the exponential map in the cylinder, we consider the following parametrization of the cylinder of radius $R=1 / k_{n}$ from $(u, v)$ to $\mathbb{R}^{3}$ :

$\mathbf{x}(u ; v)=\left(u ; R \cos \frac{v}{R} ; R \sin \frac{v}{R}\right)$.

Let $\mathbf{x}(0 ; 0)=(0 ; R ; 0)$ be the position of the point $\Phi(\mathbf{X})$ in this parametrization. Note that this parametrization is an isometry from the plane into the cylinder. Therefore, the geodesic in the cylinder that passes through $\Phi(\mathbf{X})$ and is tangent to $\mathbf{w}$ is the image of the straight line $u(s)=(\cos \varphi) s ; v(s)=$ 


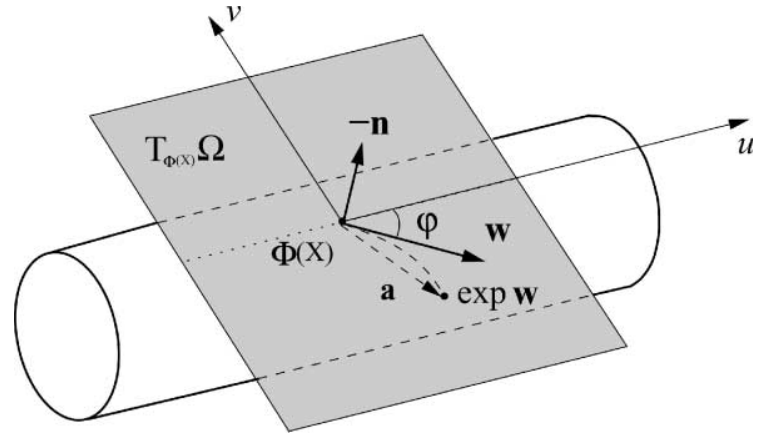

Fig. 8. The exponential map in the cylinder defined at each point of the surface by the unit normal, the normal curvature and the direction of the axis.

$(\sin \varphi) s$ parametrized by the arc length $s$ in the plane. The expression of this geodesic is:

$\mathbf{x}(s)=\left((\cos \varphi) s ; R \cos \frac{(\sin \varphi) s}{R} ; R \sin \frac{(\sin \varphi) s}{R}\right)$.

Note that this curve is a straight line if $\varphi=0 \bmod \pi$, a circle if $\varphi=\pi \bmod \pi$ and an helix otherwise. This expression allows the exponential map of $\mathbf{w}$ to be easily computed. Its components in the auxiliary coordinate system are obtained simply by evaluating the above expression at $s=A \Lambda$, the length of $\mathbf{w}$ :

$$
\begin{aligned}
& \exp _{\Phi(\mathbf{X})} \mathbf{w} \\
& \quad=\left((\cos \varphi) A \Lambda ; R \cos \frac{(\sin \varphi) A \Lambda}{R} ; R \sin \frac{(\sin \varphi) A \Lambda}{R}\right) .
\end{aligned}
$$

Finally, the deformed bond vector a is defined by the segment that goes from $\Phi(\mathbf{X})$ to $\exp _{\Phi(\mathbf{X})} \mathbf{w}$. Its components are:

$$
\begin{gathered}
\mathbf{a}=\left(A \Lambda \cos \varphi ; \frac{1}{k_{n}}\left[\cos \left(k_{n} A \Lambda \sin \varphi\right)-1\right] ;\right. \\
\left.\frac{1}{k_{n}} \sin \left(k_{n} A \Lambda \sin \varphi\right)\right) .
\end{gathered}
$$

Let us introduce for convenience the function $\mathscr{F}(x)=\sin x / x$. The deformed lattice vector can be rewritten as:

$$
\mathbf{a}=\left(p ;-\frac{k_{n} q^{2}}{2} \mathscr{F}^{2}\left(\frac{k_{n} q}{2}\right) ; q \mathscr{F}\left(k_{n} q\right)\right),
$$

where $p=A \Lambda_{1} E^{1}$ and $q=A \Lambda_{2} E^{2}$. Note that when the surface becomes planar, the argument of $\mathscr{F}(x)$ tends to zero, where this function is continuous and $\mathscr{F}(0)=1$. From a practical point of view, the computation of this function and its derivatives must be implemented carefully to prevent inaccurate results or divide by zero errors. Here, a Taylor series expansion is used when $x$ is smaller than a certain threshold.

There are three types of bonds in the graphene lattice. The formulas above are applied to each of these bond vectors $\mathbf{A}^{i}, i=1,2,3$, and it can be easily checked that the deformed lengths can be written as:

$\left\|\mathbf{a}^{i}\right\|^{2}=\frac{4}{k_{n}^{2}} \sin ^{2}\left(\frac{k_{n} q^{i}}{2}\right)+\left(p^{i}\right)^{2}$.

These three vectors form three kind of angles. The angle between two bonds $\mathbf{a}^{i}$ and $\mathbf{a}^{j}$ can be obtained as follows:

$\cos \theta^{k}=\frac{\mathbf{a}^{i} \cdot \mathbf{a}^{j}}{\left\|\mathbf{a}^{i}\right\|\left\|\mathbf{a}^{j}\right\|}$,

where $\{i, j, k\}$ is an even permutation of $\{1,2,3\}$. Thus, through an appropriate approximation of the exponential Born rule for the present kinematic setting, the deformed geometric quantities $\left\|\mathbf{a}^{i}\right\|$ and $\theta^{k}$ that occur in the atomistic potential (see Eq. (27)) have been expressed in terms of the continuum deformation, i.e. in terms of $\Lambda_{1}, \Lambda_{2}$ and $k_{n}$.

\subsection{Strain energy density}

To develop an expression for the strain energy density of the nanotube based on the inter-atomic potentials, a homogenization process at the level of the representative volume element (RVE) is carried out. The strain energy density considered is per unit undeformed area.

We argue now that the hexagonal cell of the crystal is a RVE for the closest neighbor interaction potentials considered. The area of the hexagonal cell in the undeformed graphene sheet is $S_{0}=(3 \sqrt{ } 3 / 2) A_{0}^{2}$. In each cell there is one bond of each type and two angles of each type. Indeed, it is easy to show from the expressions in the previous section that, on the one hand, the length of the 
deformed bonds corresponding to $\mathbf{A}^{i}$ and to $-\mathbf{A}^{i}$ is the same, and on the other hand the angle that the deformed bonds corresponding to $\mathbf{A}^{i}$ and $\mathbf{A}^{j}$ form is equal to the angle corresponding to $-\mathbf{A}^{i}$ and $-\mathbf{A}^{j}$. To see this, note either graphically or algebraically that the deformed bond a corresponding to an undeformed bond $\mathbf{A}$ and the deformed bond $\mathbf{a}^{-}$corresponding to $-\mathbf{A}$ are related by a symmetry with respect to the line defined by $\Phi(\mathbf{X})$ and $\mathbf{n}$.

Therefore, the hyper-elastic potential for the membrane $W$ is obtained by comparison with the energy of the deformed cell:

$$
\begin{aligned}
S_{0} \cdot W & =E_{\text {cell }} \\
& =\sum_{l=1}^{3} \mathscr{V}_{s}\left(r_{l}\right)+2 \sum_{k=1}^{3} \mathscr{V}_{\theta}\left(\theta^{k}, r_{i}, r_{j}\right),
\end{aligned}
$$

where $l$ runs over the three types of bonds, $k$ over the three types of angles, and $\{i, j, k\}$ is an even permutation of $\{1,2,3\}$. By substituting the lengths and angles of Eqs. (45) and (46), which depend on the continuum strain measures $\mathbf{C}$ and $k_{n}$, and recalling the dependence of the undeformed bond vectors on the inner displacements $\boldsymbol{\eta}$ (see Eqs. (25) and (26)), we have:

$$
\begin{aligned}
W\left(\mathbf{C}, k_{n} ; \boldsymbol{\eta}\right)=\frac{1}{S_{0}} & {\left[\sum_{l=1}^{3} \mathscr{V}_{s}\left(\left\|\mathbf{a}_{l}\right\|\right)\right.} \\
& \left.+2 \sum_{k 1}^{3} \mathscr{V}_{\theta}\left(\theta_{k},\left\|\mathbf{a}_{i}\right\|,\left\|\mathbf{a}_{j}\right\|\right)\right],
\end{aligned}
$$

where $\{i, j, k\}$ is an even permutation of $\{1,2,3\}$. Thus, the strain energy density depends not only on the local deformation of the body, but also on $\boldsymbol{\eta}$. In this continuum setting, the inner displacements should be viewed as an internal variable. The standard treatment of this internal variable is followed (Zanzotto, 1996; Tadmor et al., 1999). Given a local continuum deformation state, the energy is minimized with respect to the internal configuration:

$$
\begin{aligned}
\hat{\boldsymbol{\eta}}\left(\mathbf{C}, k_{n}\right) & =\arg \left(\min _{\boldsymbol{\eta}} W\left(\mathbf{C}, k_{n} ; \boldsymbol{\eta}\right)\right) \\
& \left.\Rightarrow \frac{\partial W}{\partial \boldsymbol{\eta}}\right|_{\hat{\boldsymbol{\eta}}\left(\mathbf{C}, k_{n}\right)}=0 .
\end{aligned}
$$

After this internal relaxation, the continuum hyper-elastic potential can be written exclusively in terms of the strain measures $\mathbf{C}$ and $k_{n}$ :

$W=W\left(\mathbf{C}, k_{n} ; \hat{\boldsymbol{\eta}}\left(\mathbf{C}, k_{n}\right)\right)=\widehat{W}\left(\mathbf{C}, k_{n}\right)$.

As noted by Tadmor et al. (1999), the derivatives of the resulting strain energy density with respect to the strain measures, for instance $\mathbf{C}$, can be computed as:

$\frac{\partial \widehat{W}}{\partial \mathbf{C}}=\frac{\partial W}{\partial \mathbf{C}}+\frac{\partial W}{\partial \eta^{i}} \frac{\partial \hat{\eta}^{i}}{\partial \mathbf{C}}$.

But, using the fact that when evaluated at the relaxed inner displacements the second part of Eq. (49) holds, we have the simpler expression:

$$
\frac{\partial \widehat{W}}{\partial \mathbf{C}}=\left.\frac{\partial W}{\partial \mathbf{C}}\right|_{\hat{\boldsymbol{\eta}}} .
$$

The same argument can be made for derivatives with respect to $k_{n}$.

If we consider a slab of cylinder of unit length, $1 \cdot \widehat{W}$ can be also viewed as a strain energy density per unit length. As shown in the following section, this potential can be appropriately handled to obtain models of reduced dimensionality analogous to the plane strain and plane stress conditions in $3 \mathrm{D}$ elasticity. The numerical examples will assess to what degree this membrane mimics accurately the atomic system. Let us now emphasize and summarize some of the properties of the theory developed:

- The continuum model for the one atom thick crystalline film is a zero-thickness membrane, in contrast with the usual shell models found in the literature.

- The hyper-elastic potential is written in closedform, i.e. no local atomistic calculations are required. The method can be viewed as constrained atomistics in which the constraints are enforced analytically.

- The strain energy density of the membrane depends on the Green deformation tensor and the curvature of the membrane. Therefore, it is frame-indifferent.

- The hyper-elastic potential inherits naturally the symmetries of the original crystal, and in 
particular, the nanotube chirality is automatically accounted for.

- This potential also accounts for local rearrangements of the Bravais multi-lattice, by treating the inner displacements.

- The continuum constitutive model has no other input than the particular molecular theory considered. Although two-body and three-body potentials have been used, the theory is not limited to this particular atomistic description.

- The presented theory applies to finite deformations. Similarly to the case of the atomic chain, the non-linear theory for the transverse deformation of nanotubes mimics exactly the atomic system for deformations with uniform strain measures. Indeed, in this case the kinematics of the exponential Born rule are exact, and therefore so is the energy.

\subsection{Non-bonded interactions and body forces}

The non-bonded or van der Waals interactions include the attractive London dispersion forces as well as the overlap repulsion forces. These interactions are usually treated by inter-atomic potentials that only act between non-bonded pairs of atoms. These diffuse interactions are critical in many applications. For the molecular system we have:

$$
\begin{aligned}
\mathscr{V}_{\text {tot,nb }} & =\sum_{i} \sum_{j>i, j \notin B_{i}} \mathscr{V}_{\mathrm{nb}}\left(r_{i j}\right) \\
& =\frac{1}{2} \sum_{i} \sum_{j \notin B_{i}} \mathscr{V}_{\mathrm{nb}}\left(r_{i j}\right),
\end{aligned}
$$

where $\mathscr{V}_{\mathrm{nb}}$ is the non-bonded potential and $B_{i}$ is the set of atoms bonded to atom $i$. The second version of the total non-bonded potential is provided because it simplifies the continuum formulation. A simple argument involving two RVEs containing two nuclei each allows us to write the continuum counterpart as:

$$
\begin{aligned}
\Pi_{\mathrm{nb}}= & \frac{1}{2} \frac{4}{S_{0}^{2}} \\
& \times \int_{\Omega_{0}} \int_{\Omega_{0}-B_{\mathbf{X}}} \mathscr{V}_{\mathrm{nb}}(\|\Phi(\mathbf{X})-\Phi(\mathbf{Y})\|) \mathrm{d} \Omega_{0 Y} \mathrm{~d} \Omega_{0 X},
\end{aligned}
$$

where $B_{\mathbf{X}}$ is a ball centered at $\mathbf{X}$ and with a radius which is function of $A_{0}$ to account for the fact that this potential does not affect bonded atoms. Finally the numerical integration version is:

$$
\begin{aligned}
\Pi_{\mathrm{nb}} & =\frac{1}{2} \frac{4}{S_{0}^{2}} \sum_{i} \sum_{j \notin B_{i}} \mathscr{V}_{\mathrm{nb}}\left(\left\|\Phi\left(\mathbf{X}_{i}\right)-\Phi\left(\mathbf{X}_{j}\right)\right\|\right) w_{i} w_{j} \\
& =\frac{4}{S_{0}^{2}} \sum_{i} \sum_{j>i, j \notin B_{i}} \mathscr{V}_{\mathrm{nb}}\left(\left\|\Phi\left(\mathbf{X}_{i}\right)-\Phi\left(\mathbf{X}_{j}\right)\right\|\right) w_{i} w_{j}
\end{aligned}
$$

where $w_{i}$ denotes the integration weights and $\mathbf{X}_{i}$ the sample points. Note that with the numerical integration, the structure of the original potential, which loops over interactions and not twice over the domain, is recovered.

When external forces are applied on the nuclei (e.g. electrostatic forces), the continuum counterpart is a body force, and the corresponding external potential is:

$\Pi_{\text {ext }}=\int_{\Omega_{0}} \mathbf{B} \cdot \Phi \mathrm{d} \Omega_{0}$,

where $\mathbf{B}$ is the body force per unit undeformed area. If the forces applied on the atomic system are a certain constant force $\mathbf{f}$ acting on each atom, then $\mathbf{B}$ is simply given by the expression:

$\mathbf{B}=\frac{2}{S_{0}} \mathbf{f}$.

\subsection{Variational principle and stress measures}

Considering the bonded potentials, the nonbonded interactions and the body forces, the total potential energy of a given deformation map $\Psi$ is:

$$
\begin{aligned}
& \Pi(\Psi)=\Pi_{\mathrm{int}}-\Pi_{\mathrm{ext}}+\Pi_{\mathrm{nb}} \\
& =\int_{\Omega_{0}} \widehat{W}\left(\mathbf{C}(\Psi), k_{n}(\Psi)\right) \mathrm{d} \Omega_{0} \\
& -\int_{\Omega_{0}} \mathbf{B} \cdot \Psi \mathrm{d} \Omega_{0}+\frac{1}{2} \frac{4}{S_{0}^{2}} \\
& \times \int_{\Omega_{0}} \int_{\Omega_{0}-B_{\mathbf{X}}} \mathscr{V}_{\mathrm{nb}}(\|\Psi(\mathbf{X})-\Psi(\mathbf{Y})\|) \mathrm{d} \Omega_{0 Y} \mathrm{~d} \Omega_{0 X} .
\end{aligned}
$$


The continuum statement of the problem of finding stable equilibrium solutions is then given by:

$$
\Phi=\arg \left[\inf _{\Psi \in \mathscr{C}} \Pi(\Psi)\right],
$$

where $\mathscr{C}$ is the appropriate space of deformations or trial functions accounting for essential boundary conditions. According to the principle of stationary energy, the equilibrium solutions of the system are stationary points of the potential energy functional, and they verify the principle of virtual work:

$$
\begin{aligned}
0= & \delta \Pi(\Phi) \\
= & \left.\int_{\Omega_{0}} \frac{\partial \widehat{W}}{\partial \mathbf{C}}: \delta \mathbf{C}+\frac{\partial \widehat{W}}{\partial k_{n}} \delta k_{n}\right) \mathrm{d} \Omega_{0} \\
& -\int_{\Omega_{0}} \mathbf{B} \cdot \delta \Phi \mathrm{d} \Omega_{0}+\delta \Pi_{\mathrm{nb}},
\end{aligned}
$$

where $\delta \Phi$ denotes the virtual deformation. The variations of the non-bonded continuum potential can be written as:

$$
\begin{aligned}
\delta \Pi_{\mathrm{nb}}= & \frac{1}{2} \frac{4}{S_{0}^{2}} \int_{\Omega_{0}} \int_{\Omega_{0}-B_{\mathbf{X}}} \frac{\mathscr{V}_{\mathrm{nb}}^{\prime}}{\|\Phi(\mathbf{X})-\Phi(\mathbf{Y})\|} \\
& \times[\Phi(\mathbf{X})-\Phi(\mathbf{Y})] \\
& \cdot[\delta \Phi(\mathbf{X})-\delta \Phi(\mathbf{Y})] \mathrm{d} \Omega_{0 Y} \mathrm{~d} \Omega_{0 X} .
\end{aligned}
$$

Let us also define the stress measures, always evaluated at the relaxed inner displacements $\hat{\boldsymbol{\eta}}$. Recalling the expression of the strain energy density in Eq. (48) and following a similar rationale to that used to obtain Eq. (52), we obtain:

$$
\begin{aligned}
\mathbf{S}=2 & \frac{\partial \widehat{W}}{\partial \mathbf{C}}=2 \frac{\partial W}{\partial \mathbf{C}} \\
=\frac{2}{S_{0}} & {\left[\sum_{l 1}^{3} \mathscr{V}_{s}^{\prime} \frac{\partial\left\|\mathbf{a}_{l}\right\|}{\partial \mathbf{C}}+2 \sum_{k}^{3}\left(\frac{\partial \mathscr{V}_{\theta}}{\partial \theta} \frac{\partial \theta_{k}}{\partial \mathbf{C}}\right.\right.} \\
& \left.\left.+\frac{\partial \mathscr{V}_{\theta}}{\partial r^{1}} \frac{\partial\left\|\mathbf{a}_{i}\right\|}{\partial \mathbf{C}}+\frac{\partial \mathscr{V}_{\theta}}{\partial r^{2}} \frac{\partial\left\|\mathbf{a}_{j}\right\|}{\partial \mathbf{C}}\right)\right]
\end{aligned}
$$

and

$$
\begin{aligned}
m=\frac{\partial \widehat{W}}{\partial k_{n}} & =\frac{\partial W}{\partial k_{n}} \\
=\frac{1}{S_{0}} & {\left[\sum_{l 1}^{3} \mathscr{V}_{s}^{\prime} \frac{\partial\left\|\mathbf{a}_{l}\right\|}{\partial k_{n}}+2 \sum_{k}^{3}\left(\frac{\partial \mathscr{V}_{\theta}}{\partial \theta} \frac{\partial \theta_{k}}{\partial k_{n}}\right.\right.} \\
& \left.\left.+\frac{\partial \mathscr{V}_{\theta}}{\partial r^{1}} \frac{\partial\left\|\mathbf{a}_{i}\right\|}{\partial k_{n}}+\frac{\partial \mathscr{V}_{\theta}}{\partial r^{2}} \frac{\partial\left\|\mathbf{a}_{j}\right\|}{\partial k_{n}}\right)\right] .
\end{aligned}
$$

The in-plane stress $\mathbf{S}$ corresponds precisely to the Second Piola Kirchhoff stress, while $m$ is a moment-like stress. Note that, because of the special form of $\mathbf{C}$ (see Eq. (33)), $\mathbf{S}$ has only two non-zero components, which are related to the tractions in the axial and the circumferential directions. On the other hand, $m$ is here a scalar. The general theory for arbitrary deformations is given in Arroyo and Belytschko (2002). Note that, since the membrane has no thickness, the units of $\mathbf{S}$ are force divided by length, while $m$ is expressed in units of force (bending moment divided by length).

Using the Green strain tensor $\mathbf{E}=1 / 2(\mathbf{C}-\mathbf{I})$, we can rewrite the principle of virtual work as:

$$
\begin{aligned}
0= & \int_{\Omega_{0}}\left(\mathbf{S}: \delta \mathbf{E}+m \delta k_{n}\right) \mathrm{d} \Omega_{0} \\
& -\int_{\Omega_{0}} \mathbf{B} \cdot \delta \Phi \mathrm{d} \Omega_{0}+\delta \Pi_{\mathrm{nb}} .
\end{aligned}
$$

Depending on the treatment of the axial stretch $\Lambda_{1}$ (see Eq. (30)) different situations can be studied:

Plane strain: We can consider the situation in which the value of $\Lambda_{1}$ is prescribed. In this case, the unknowns of the variational problem (64) are $\Phi^{2}$ and $\Phi^{3}$ (see Eq. (30)). If $\Lambda_{1}=1$, a deformation analogous to plane strain conditions is achieved. This applies to very long or axially constrained nanotubes. $\Lambda_{1}$ can also be prescribed an arbitrary value to study the transverse behavior of stretched or compressed nanotubes; the behavior will change due to the non-linearity of the model. In this situation, $\delta \Lambda_{1}=0$ and the axial component of the membrane stress does not appear in the variational principle. This means that the axial stress can be computed a posteriori, but does not play a role in the solution of the problem.

Plane stress: Alternatively, the axial component of the membrane stress can be prescribed, for in- 
stance, to be zero. This would be the case of axially unconstrained nanotubes. In this case, in addition to $\Phi^{2}$ and $\Phi^{3}$, the axial stretch $\Lambda_{1}$ becomes an unknown of the problem.

\section{Validation and representative simulations}

In this section, numerical simulations of stable configurations of carbon nanotubes in different situations are reported. The reduced continuum model described in the previous section is used and the variational principle described in Eq. (64) is discretized by Galerkin finite elements (FE). Thus, the original discrete molecular system is replaced by a continuum model which is subsequently transformed by the FE method into another discrete system. However, in principle we are free to design the FE discretization so that the FE model has fewer degrees of freedom than the original system. Furthermore, since the continuum model is $2 \mathrm{D}$, while the full atomistic model is $3 \mathrm{D}$, the computational cost is further reduced.

First, the exponential Born rule-based continuum model is validated by comparing FE simulations based on it with full atomistic calculations. In these comparisons the inter-atomic potentials used in the MM simulations are used to construct the continuum constitutive equation, and analogous boundary conditions are considered in both calculations. Since the continuum model is intended to mimic the atomistic system, which is viewed as "true", the term error should be understood as deviation form the atomistic model. Simulations show that the agreement is excellent with regard to the energetics as well as to the stable configurations. Simulations of a model based on the standard Born rule are also provided, illustrating the deficiencies of such a model. The exponential Born rule simulations also show that, for the tested situations, the relaxation of the inner displacements greatly affects the energetics but has very little impact on the minimum energy configurations. The continuum model is then applied to simulate several situations where the transverse behavior of carbon nanotubes and the effect of van der Waals interactions are important. A final ex- ample of the generalization of the model to three dimensions is presented, with a twisting test of a $[10,10]$ nanotube beyond the point of structural instabilities.

The inter-atomic potentials fall into the general form described in Eq. (28). The two-body potential $\mathscr{V}_{s}$ is a Morse potential while the three-body potential depends only on the angle $\mathscr{V}_{\theta}$ and is harmonic with a sextic correction. The parameters are taken from the MM2 model. The non-bonded interactions are based on the classical LennardJones (6 12) potential.

The variational principle in Eq. (64) imposes restrictions on the finite element interpolation spaces. The virtual internal work term involves variations on the curvature of the test functions, and therefore the finite element space needs to be $H^{2}$, i.e. have up to second square integrable derivatives. This is why $\mathscr{C}^{1}$ Hermite finite elements are chosen. Note that the discretization of the configuration described in Eq. (30) requires the approximation of the scalar functions $\Phi^{2}(\cdot)$ and $\Phi^{3}(\cdot)$, i.e. the curve in $\mathbb{R}^{2}$ described by these functions needs to be parametrized with respect to the finite element degrees of freedom. Each of these functions is approximated by piecewise $\mathscr{C}^{1}$ cubic polynomials, and therefore, each node $I$ carries four degrees of freedom: $\Phi_{I}^{2}, \Phi_{I}^{3},\left(\Phi^{2}\right)_{I}^{\prime}$ and $\left(\Phi^{3}\right)_{I}^{\prime}$. The internal and external work terms of the variational principle are integrated using 3 Gauss points per element, while the integration of the non-bonded interactions term may require more integration points depending on the size of the finite elements relative to the van der Waals equilibrium distance. Four integration points are required for this term in some of the simulations.

The BFGS quasi-Newton technique is used both in the relaxation of the inner displacements and in the global energy minimization. This iterative method only requires gradients of the objective function and approximates the inverse of its Hessian using information from the previous iterations. For some of the larger examples involving more than one nanotube, and when the initial configuration is very far from equilibrium, dynamical relaxation is used to obtain a good first guess which is further refined with the BFGS minimization algorithm. 


\subsection{Validation test}

To validate the proposed reduced continuum model, a FE discretized version is compared to a MM model. A [32,0] zigzag carbon nanotube (the standard description of carbon nanotubes in terms of two integers is described by Saito et al. (1992)) is considered (see Fig. 9(a)). The molecular model used in the comparison has 384 nuclear positions, that is 1152 degrees of freedom, while the FE model has 20 nodes and consequently 80 degrees of freedom. Note that the discrete FE model reduces the computational cost, not only because large elements relative to the crystal cell size can be used, but also because of its reduced dimensionality.

The first configuration studied consists of simply rolling a graphene sheet into a tube in an isometric transformation, without any kind of relaxation. This configuration is called Original tube in Table 1. The table shows the excellent agreement between the energy obtained with the molecular model and that obtained via the continuum model and FE. According to the last remark of Section 6.2, the energy of the continuum model should be exact in this situation. Note however that the continuum membrane is discretized using an approximation space that does not reproduce exactly a circle, and thus introduces discretization errors.

Then several "plane strain" situations are considered. This condition is enforced in the molecular model by prescribing to zero the nuclear displacements in the direction of the axis of the tube at the nuclei located at both ends of the tube. In the continuum model, we simply enforce $\Lambda_{1}=1$.

Also, two kinds of energy minimization are considered. The first one freezes the inner displacements to those of the graphene sheet in equilibrium, i.e. in the continuum model by prescribing $\boldsymbol{\eta}=0$. In this particular example, invoking symmetry considerations, this constrained minimization can be easily implemented in the molec-
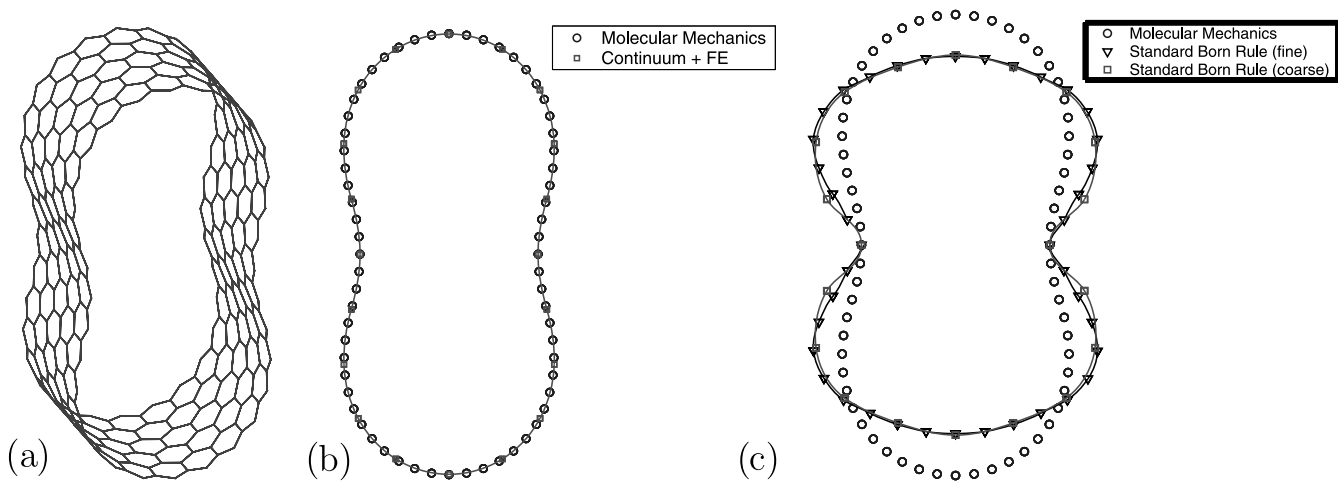

Fig. 9. (a) Actual molecular model used in comparison, (b) comparison of 20 element exponential Born rule continuum model with MM and (c) results obtained with a model constructed from the standard Born rule.

Table 1

Comparison of 20 element model $(C+\mathrm{FE})$ with MM: energy in $\mathrm{J} / \mathrm{mol}$

\begin{tabular}{|c|c|c|c|c|c|c|}
\hline & \multicolumn{3}{|l|}{$\begin{array}{ll}\eta & 0 \\
\end{array}$} & \multicolumn{3}{|c|}{ Relaxed $\boldsymbol{\eta}$} \\
\hline & $\mathrm{MM}$ & $C+\mathrm{FE}$ & Error $(\%)$ & $\mathrm{MM}$ & $C+\mathrm{FE}$ & Error $(\%)$ \\
\hline Original tube & 14.58 & 14.69 & 0.81 & & & \\
\hline Relaxed tube & 10.26 & 10.28 & 0.22 & 6.338 & 6.324 & 0.22 \\
\hline Squeezed A & 20.85 & 21.22 & 1.8 & 12.95 & 13.11 & 1.2 \\
\hline Squeezed B & 48.56 & 49.17 & 1.3 & 30.68 & 30.46 & 0.75 \\
\hline
\end{tabular}


ular model by prescribing to zero the displacements of all the nuclei in the direction of the tube axis. This incomplete analysis is performed to highlight the effect of the inner relaxation. The other analysis is an unconstrained structural optimization of all the nuclear positions. In the continuum, the inner displacements are relaxed in order to calculate $\widehat{W}$ at each Gauss point.

The situations considered are:

Relaxed tube: The Original tube is relaxed without any constraint other than the plane strain conditions.

Squeezed A: Displacements at the ends of one diameter of the tube are prescribed so that this diameter of is squeezed to $3 / 4$ of its original size.

Squeezed B: Displacements at the ends of one diameter of the tube are prescribed so that this diameter of is squeezed to $1 / 2$ of its original size.

Table 1 presents the equilibrium energies for both the MM and the Continuum FE simulations, as well as the relative error of the FE calculation with respect to MM. A positive value of error means that the MM energy is lower than the FE energy. Note that this error includes contributions not only from the modelling of the discrete atomic system as a membrane, but also from the FE discretization.

Fig. 9(b) compares the equilibrium configurations for the continuum/FE model and the MM model in the Squeezed B situation. Despite the large deformations to which the tube is subjected, the agreement is excellent. Table 1 shows that the equilibrium energies obtained with the continuum model are in all the cases very accurate approximations of the MM energies. The discrepancies are in all the cases below $2 \%$. The effect of the inner relaxation in the magnitude of the energies is very important. In this table 20 finite elements have been used, while 32 hexagonal cells span the same perimeter in the MM model. Therefore, we expect the FE model to be more constrained and therefore yield higher equilibrium energies. This can be noticed in the columns corresponding to frozen inner displacements. However, when those are relaxed, the FE model reaches lower energies than the molecular model, still remaining very accurate. Probably the continuum treatment of the inner displacements allows for this extra relaxation.

Although the effect of the inner relaxation in the equilibrium energies is very important, in these simulations its effect on the stable configurations is negligible. This can be explained by noting that the in-plane behavior of the model is very stiff, while the flexural behavior is very compliant. Therefore, a slight perturbation of in-plane deformation (the inner rearrangements are an in-plane effect) has dramatic influence on energy, but not in these flexural-dominated optimal deformations. This suggests that in these examples, the inner relaxation is nearly uncoupled from the bending deformation. This is not the case for other types of deformation (Arroyo and Belytschko, 2002).

Table 2 shows the results obtained with 36 finite elements. The errors obtained are smaller in all the cases except in the Squeezed B situation with inner relaxation. This indicates that in general the richer discretization decreases the overall error, but also that the finer mesh allows other modeling errors to manifest themselves. Indeed, the error probably increases in the last case because the continuum model is more compliant than the molecular one with regard to the inner displacements. However, simulations carried out with even finer meshes indicate that the results "converge" to a very accurate result. Thus, even if the FE model is refined beyond the unit cell size, the continuum model

Table 2

Comparison of 36 element model $(C+\mathrm{FE})$ with MM: energy in $\mathrm{J} / \mathrm{mol}$

\begin{tabular}{|c|c|c|c|c|c|c|}
\hline & \multicolumn{3}{|l|}{$\begin{array}{ll}\eta \quad 0 \\
\end{array}$} & \multicolumn{3}{|c|}{ Relaxed $\boldsymbol{\eta}$} \\
\hline & $\mathrm{MM}$ & $C+\mathrm{FE}$ & Error $(\%)$ & $\mathrm{MM}$ & $C+\mathrm{FE}$ & Error $(\%)$ \\
\hline Original tube & 14.58 & 14.60 & 0.14 & & & \\
\hline Relaxed tube & 10.26 & 10.28 & 0.21 & 6.338 & 6.324 & 0.22 \\
\hline Squeezed A & 20.85 & 21.05 & 0.96 & 12.95 & 12.99 & 0.31 \\
\hline Squeezed B & 48.56 & 49.01 & 0.93 & 30.68 & 30.34 & 1.1 \\
\hline
\end{tabular}



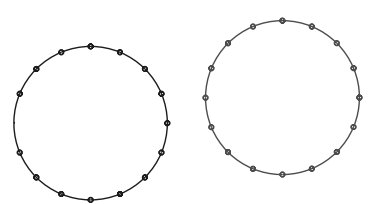

$[20,0]$ tube; $\Delta \mathrm{E}=0$

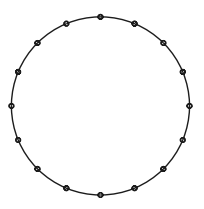

$[32,0]$ tube; $\Delta \mathrm{E}>0$

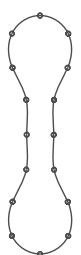

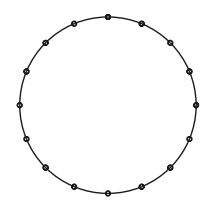

$[26,0]$ tube; $\Delta \mathrm{E}<0$
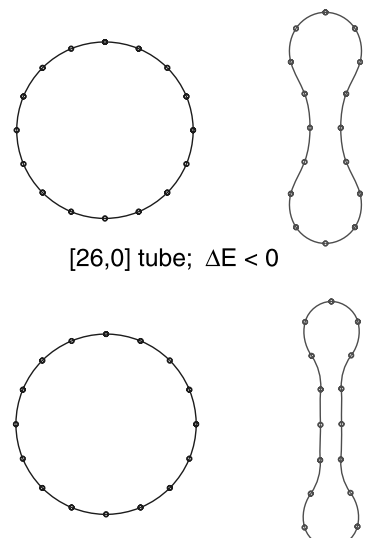

$[40,0]$ tube; $\Delta \mathrm{E}>0$

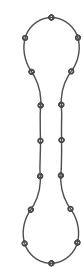

Fig. 10. Which is more stable, circular or collapsed? (Answer: for the $[20,0]$ and $[26,0]$ tubes, circular, and for the $[32,0]$ and $[40,0]$ tubes, collapsed.)
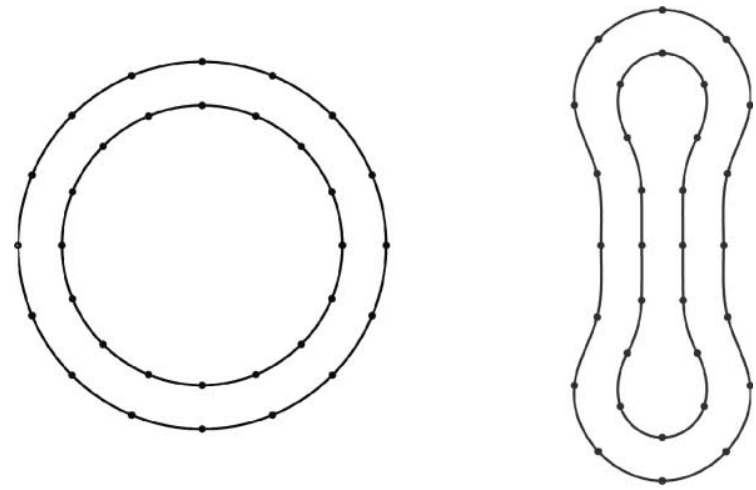

$[42,0]$ and $[32,0]$ nested tubes; $\Delta \mathrm{E}>0$

Fig. 11. Transverse stability of a multi walled nanotube.

apparently does not exhibit fine features that cannot be present in the molecular model.

This excellent behavior contrasts with the situation encountered when a continuum model for the membrane is directly constructed from the Born rule without the proposed exponential extension. In this case the resulting hyper-elastic potential is non-convex. Indeed, as discussed in Section 4.3 , the energy of such a model is invariant under isometric deformations (bending without stretch), i.e. the model has zero bending stiffness. This reflects in a pathological mesh dependency in
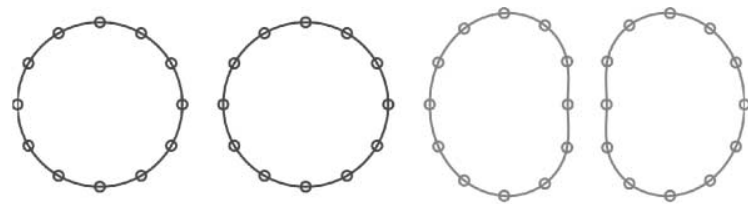

Two $[20,0]$ nanotubes
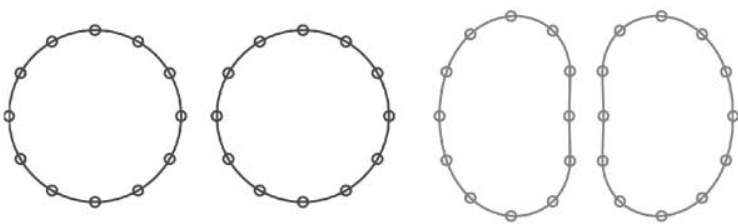

Two $[24,0]$ nanotubes
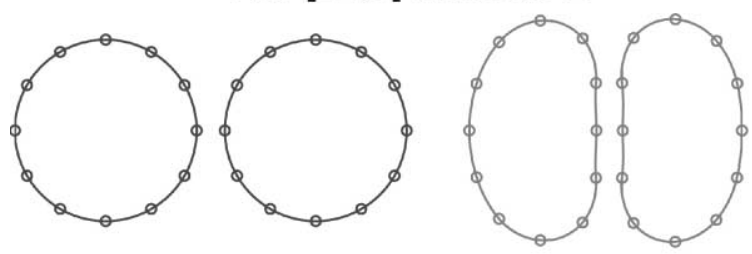

Two [32,0] nanotubes

Fig. 12. Equilibrium configurations for pairs of nanotubes in van der Waals contact.
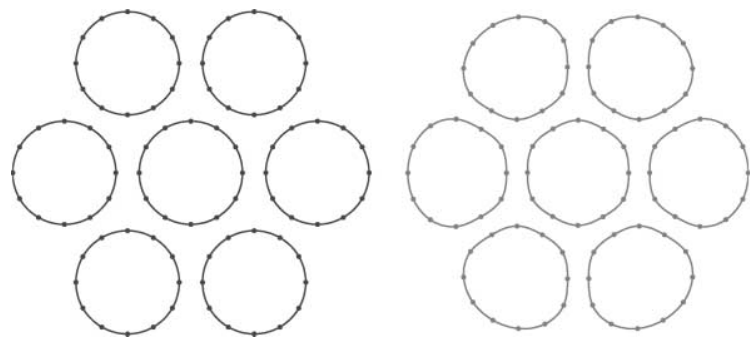

Fig. 13. Equilibrium configuration of a bundle of seven closely packed $[22,0]$ nanotubes.

the numerical implementation of such a model: since the discrete FE space cannot represent all isometric deformations, the discrete problem can still be solved, but as the mesh is refined, the numerical method picks solutions with increasingly finer features. Fig. 9(c) illustrates this fact, and sharper kinks in the numerical solution are observed as the mesh is refined. The equilibrium energy of the FE solutions is almost zero, which is not realistic. This is reminiscent of the situation encountered in other materials, for which the 


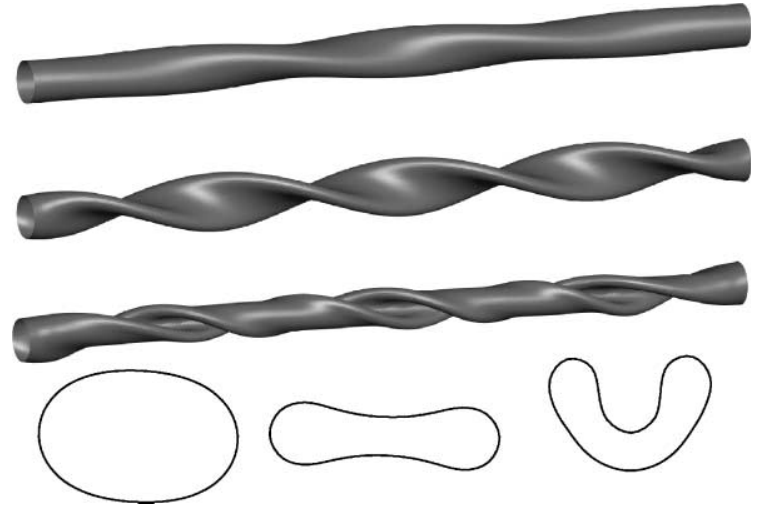

Fig. 14. Twisting of a $[10,10]$ nanotube: deformed geometry for twisting angles of $38^{\circ}, 210^{\circ}$ and $360^{\circ}$, and cross section of the deformed membrane at the center of the tube for the above three configurations.

strain energy density is physically con-convex, leading to non-unique solutions with increasingly fine features, as reported by Dacorogna (1989, p. 276) and references therein.

\subsection{Transverse deformation simulations}

The next simulations illustrate the application of the continuum/FE model to the transverse mechanics of nanotubes in different situations. In these applications, the computational cost of analogous MM simulations would be much higher than the cost of the presented calculations. This is especially true with regards to the non-bonded interactions.

The first example studies the stability of the circular and the collapsed configurations of carbon nanotubes. Because of the van der Waals attraction potential, the energy of the system is reduced when two walls adhere. On the other hand, for the wall of a nanotube to come in contact with itself, significant elastic energy is required. This tradeoff is probably responsible for the observation by Gao et al. (1998) that below a certain radius, only the circular configuration is stable. For greater radii, the collapsed configuration is at least meta-stable. Subsequently, another threshold radius separates the nanotubes for which the circular configuration is energetically favorable from those in which the collapsed configuration is. Fig. 10 shows the sim- ulations performed for several nanotubes. In this and subsequent figures, the nodes shown are nodes of the finite element mesh; they are not atoms. In these calculations, the fully relaxed circular configuration is deformed so that the wall of the nanotube is brought in contact with itself at the van der Waals equilibrium distance, and then the energy is minimized. The sign of the difference in energy between the circular configuration and the relaxed configuration is also reported, i.e. a positive difference means that the energy of the configuration presented on the right is lower. In some cases, the nanotube goes back to the original configuration (this is the case of the $[20,0]$ nanotube). This implies that the collapsed configuration is not stable. The collapsed configuration is stable for the $[26,0]$ nanotube, but this only constitutes a local minimum of the energy since the circular configuration has lower energy. For the $[32,0]$ and $[40,0]$ nanotubes, the collapsed configuration is the energetically favorable structure. This is expected because larger nanotubes are more flexible and have more wall area to gain adhesion energy. Fig. 11 displays a similar analysis for a multi-walled nanotube for which the collapsed configuration yields lower energy .

A similar competition of elastic and adhesion energy occurs when two nanotubes are brought to the van der Waals equilibrium distance. Fig. 12 shows the equilibrium configurations obtained when this numerical experiment is performed with nanotubes of different sizes. Again, the larger nanotubes have larger portions of flattened walls.

We also report a simulation of a bundle of nanotubes under plane strain. Fig. 13 shows the equilibrium configuration of the system. A TEM image of such a nanorope has been reported by Salvetat et al. (1999). Carbon nanotubes tend to be closely packed in hexagonal lattices in the nanoropes and crystals of nanotubes (Thess et al., 1996; Schlittler et al., 2001). As can be seen from Fig. 13, the equilibrium configuration displays a flattening of the nanotube walls, or partial polygonalization.

\subsection{Three dimensional simulation}

The theory presented has been used to construct a membrane applicable in the general 3D 
deformation of carbon nanotubes (Arroyo and Belytschko, 2002). This more general membrane can be discretized with subdivision finite elements, and the structural instabilities reported in experiments and atomistic simulations can be analyzed at very low computational cost. The analysis of twisting a $[10,10]$ nanotube is provided in Fig. 14, for a Tersoff-Brenner potential. Note that, the deformed geometries have been post-processed, and the computational mesh has about 18 elements around the perimeter. Each end of the nanotube is incrementally rotated $360^{\circ}$ in opposite orientations.

The first snapshot of the deformation shows the configuration when the first instability from a uniform twisting occurs, and the corresponding cross-section is shown at the bottom of the figure. Further twisting causes the wall of the nanotube to come in van der Waals contact with itself, as clearly shown in the cross-section in the bottom of Fig. 14. Beyond $210^{\circ}$, a secondary instability develops, and the tube folds onto itself. From the cross-section it is apparent that the van der Waals interactions are responsible for this buckled morphology. In the absence of these long-range forces, the membrane inter-penetrates and the secondary structure is not observed. This $3 \mathrm{D}$ membrane has been shown to provide very accurate energetics and deformed geometries even for very large deformations (Arroyo and Belytschko, 2002).

\section{Conclusions}

We have further explored a methodology to construct continuum models for one-atom thick crystalline films. The proposed model is a hyperelastic membrane whose elastic potential energy is written in closed-form exclusively in terms of the inter-atomic potentials that constitute the molecular description of the system. The analysis of the present work is based on the exponential the Born rule (Arroyo and Belytschko, 2002), a kinematic assumption linking the atomic and the continuum deformations when the crystal is a curved film. This extension is based on the exponential map. An illustrative example of an atomic chain deforming in two dimensions has been presented. The resulting simple rope-like continuum model encompasses all of the fundamental ideas.

The general methodology then is particularized to analyze the transverse mechanics of carbon nanotubes. This model explicitly exploits the symmetry of such a deformation, and leads to a model of reduced dimensionality. The hyper-elastic potential, as well as strain and stress measures are provided, and a continuum formulation of the non-bonded interactions is derived. The proposed model is discretized using finite elements, yielding an alternative simulation method that is faster than atomistic calculations.

Several simulations highlighting the relevance of van der Waals interactions in the transverse mechanics of nanotubes are reported. The results show that the continuum model based on the exponential Born rule very well approximates the stable configurations and energies of the corresponding MM model. Results agree with MM calculations within $2 \%$ in the equilibrium energies. This sharply contrasts with the non-physical results obtained from a model based on the standard Born rule. We also show the important effect of the inner rearrangements of the crystal structure on the equilibrium energies. A full 3D simulation illustrates the application of the present theory to analyze the structural instabilities of nanotubes observed in experiments and atomistic calculations.

\section{Acknowledgements}

The support of the "la Caixa" Graduate Program to M. Arroyo, and the National Science Foundation and the U.S. Army Research Office is gratefully acknowledged.

\section{References}

Arroyo, M., Belytschko, T., 2002. An atomistic based finite deformation membrane for single layer crystalline films. Journal of the Mechanics and Physics of Solids 50, 1941 1977.

Bernholc, J., Brabec, C.J., Nardelli, M.B., Maiti, A., Roland, C., Yakobson, B.I., 1998. Theory of growth and mechanical properties of nanotubes. Applied Physics A 67, 3946. 
Brenner, D.W., 1990. Empirical potential for hydrocarbons for use in simulating chemical vapor deposition of diamond films. Physical Review B 42 (15), 94589471.

Chopra, N.G., Benedict, L.X., Crespi, V.H., Cohen, M.L., Louie, S.G., Zettl, A., 1995. Fully collapsed carbon nanotubes. Nature 377, 135138.

Cousins, C.S.G., 1978. Inner elasticity. Journal of Physics C, 48674879.

Dacorogna, B., 1989. Direct methods in the calculus of variations. In: Applied Mathematical Sciences, vol. 78. Springer Verlag, Berlin.

do Carmo, M.P., 1976. Differential geometry of curves and surfaces. Prentice Hall, Englewood Cliffs, NJ.

Ericksen, J.L., 1984. Phase transformations and material instabilities in solids. In: Gurtin, M.E. (Ed.), The Cauchy and Born Hypotheses for Crystals. Academic Press, Lon don, pp. 6177.

Falvo, M.R., Clary, G.J., Taylor, R.M., Chi, V., Brooks, F.P., Washburn, S., Superfine, R., 1997. Bending and buckling of carbon nanotubes under large strain. Nature 389, 582584.

Friesecke, G., James, R.D., 2000. A scheme for the passage from atomic to continuum theory for thin films, nanotubes and nanorods. Journal of the Mechanics and Physics of Solids 48, 15191540.

Gao, G., Çağin, T., Goddard III, W., 1998. Energetics, structure, mechanical and vibrational properties of single walled carbon nanotubes. Nanotechnology 9, 184191.

Lu, J.P., 1997. Elastic properties of carbon nanotubes and nanoropes. Physical Review Letters 79 (7), 12971300.

Maiti, A., 2000. Mechanical deformation in carbon nanotubes bent tubes vs tubes pushed by atomically sharp tips. Chemical Physical Letters 331, 2125.

Malvern, L.E., 1969. Introduction to the mechanics of a continuous medium. Prentice Hall, Englewood Cliffs, NJ.

Marsden, J.E., Hughes, T.J., 1983. Mathematical foundations of elasticity. Prentice Hall, Englewood Cliffs, NJ.

Martin, J.W., 1975. Many body forces in metals and the brug ger elastic constants. Journal of Physics C 8, 28372857.

Morgan, F., 1993. Riemannian geometry, a beginner's guide. Jones and Bartlett Publishers.

Nevins, N., Chen, K., Allinger, N.L., 1996. Moleculas mechan ics (MM4) calculations on alkenes. Journal of Computa tional Chemistry 17 (5 6), 669694.

Qian, D., Liu, W.K., Ruoff, R.S., 2001. Mechanics of $\mathrm{C}_{60}$ in nanotubes. Journal of Physical Chemistry B 105, 10753 10758 .

Ruoff, R.S., Tersoff, J., Lorents, D.C., Subramoney, S., Chan, B., 1993. Radial deformation of carbon nanotubes by van der Waals forces. Nature 364, 514516.

Saito, R., Fujita, M., Dresselhaus, G., Dresselhaus, M.S., 1992. Electronic structure of chiral graphene tubules. Applied Physics Letters 60 (18), 22042206.

Salvetat, J.P., Briggs, G.A.D., Bonard, J.M., Bacsa, R.R., Kulik, A.J., Stockli, T., Burnham, N.A., Forró, L., 1999.
Elastic and shear moduli of single walled carbon nanotube ropes. Physical Review Letters 82 (5), 944947.

Schlittler, R.R., Seo, J.W., Gimzewski, J.K., Durkan, C., Saifullah, M.S.M., Welland, M.E., 2001. Single crystals of single walled carbon nanotubes formed by self assembly. Science 292, 11361139.

Shenoy, V.B., Miller, R., Tadmor, E.B., Rodney, D., Phillips, R., Ortiz, M., 1999. An adaptive finite element approach to atomic scale mechanics the quasicontinuum method. Journal of the Mechanics and Physics of Solids 47, 611 642.

Sohlberg, K., Sumpter, B.G., Tuzun, R.E., Noid, D.W., 1998. Continuum methods of mechanics as a simplified approach to structural engineering of nanostructures. Nanotechnol ogy 9,3036 .

Tadmor, E.B., Ortiz, M., Phillips, R., 1996. Quasicontinuum analysis of defects in solids. Philosophical Magazine A 73 (6), 15291563.

Tadmor, E.B., Smith, G.S., Bernstein, N., Kaxiras, E., 1999. Mixed finite element and atomistic formulation for complex crystals. Physical Review B 59 (1), 235245.

Thess, A., Lee, R., Nikolaev, P., Dia, H., Petit, P., Robert, J., Xu, C., Lee, Y.H., Kim, S.G., Rinzler, A.G., Colbert, D.T., Scuseria, G.E., Tománek, D., Fischer, J.E., Smalley, R.E., 1996. Crystalline ropes of metallic carbon nanotubes. Science 273, 483487.

Weiner, J.H., 1983. Statistical mechanics of elasticity. Wiley, New York.

Yakobson, B.I., Brabec, C.J., Bernholc, J., 1996. Nanome chanics of carbon tubes: Instabilities beyond the linear response. Physical Review Letters 76 (14), 25112514.

Yu, M., Dyer, M.J., Ruoff, R.S., 2001a. Structure and mechanical flexibility of carbon nanotube ribbons: An atomic force microscopy study. Journal of Applied Physics 89 (8), 45544557.

Yu, M., Kowalewski, T., Ruoff, R.S., 2001b. Structural analysis of collapsed, and twisted and collapsed, multiwalled carbon nanotubes by atomic force microscopy. Physical Review Letters 86 (1), 8790.

Yu, M., Lourie, O., Dyer, M.J., Moloni, K., Kelly, T.F., Ruoff, R.S., 2000. Strength and breaking mechanism of multi walled carbon nanotubes under tensile load. Science 287, 637640 .

Zanzotto, G., 1996. The Cauchy Born hypothesis, nonlinear elasticity and mechanical twinning in crystals. Acta Crys tallographica A 52, 839849.

Zhong Can, O. Y., Su, Z. B., Wang, C. L., 1997. Coil forma tion in multishell carbon nanotubes: competition between curvature elasticity and interlayer adhesion. Physical Re view Letters 78 (21), 40554058.

Zhou, G., Duan, W., Gu, B., 2001. First principles study on morphology and mechanical properties of single walled carbon nanotube. Chemical Physical Letters 333, 344 349. 